\title{
Drug Repurposing: Deferasirox Inhibits the Anti-Apoptotic Activity of Mcl-I
}

\author{
Asma Bourafai-Aziez, (D) \\ Mohammed Benabderrahmane, ${ }^{2}$ \\ Hippolyte Paysant, (1D ${ }^{3,4}$ \\ Louis-Bastien Weiswald, ${ }^{3,4}$ \\ Laurent Poulain, ${ }^{3,4}$ Ludovic \\ Carlier, ${ }^{5}$ Delphine Ravault, ${ }^{5}$ \\ Marie Jouanne, ${ }^{2}$ Gaël \\ Coadou, (D) 'Hassan Oulyadi,' \\ Anne-Sophie Voisin-Chiret, ${ }^{2}$ \\ Jana Sopková-de Oliveira \\ Santos, ${ }^{2}$ Muriel Sebban (D) \\ 'Normandie Université, UNIROUEN, \\ INSA de Rouen, CNRS Laboratoire \\ COBRA (UMR 60I4 \& FR 3038), Rouen, \\ 76000, France; ${ }^{2}$ Normandie Univ, \\ UNICAEN, CERMN, Caen, I4000, \\ France; ${ }^{3}$ Normandie Université, \\ UNICAEN, Inserm UI086 ANTICIPE \\ «Interdisciplinary Research Unit for \\ Cancer Prevention and Treatment», \\ Biology and Innovative Therapeutics for \\ Ovarian Cancers Group (BioTICLA), \\ Centre de Lutte Contre le Cancer F. \\ Baclesse, Caen, 14076, France; \\ ${ }^{4}$ UNICANCER, Centre de Lutte Contre \\ le Cancer F. Baclesse, Caen, I4076, \\ France; ${ }^{5}$ Sorbonne Université, École \\ Normale Supérieure, PSL University, \\ CNRS, Laboratoire des Biomolécules, \\ LBM, Paris, France
}

Correspondence: Muriel Sebban Normandie Université, UNIROUEN, INSA de Rouen, CNRS Laboratoire COBRA (UMR 60I4 \& FR 3038), Rouen, 76000 , France,

Tel +33235522944

Fax +33235522441

Email muriel.sebban@univ-rouen.fr

Jana Sopková-de Oliveira Santos Normandie Univ, UNICAEN, CERMN,

Caen, 14000, France

Tel +33231566821

Fax +33231566803

Email jana.sopkova@unicaen.fr
Introduction: With the aim of repositioning commercially available drugs for the inhibition of the anti-apoptotic myeloid cell leukemia protein, Mcl-1, implied in various cancers, five molecules, highlighted from a published theoretical screening, were selected to experimentally validate their affinity toward Mcl-1.

Results: A detailed NMR study revealed that only two of the five tested drugs, Torsemide and Deferasirox, interacted with Mcl-1. NMR data analysis allowed the complete characterization of the binding mode of both drugs to Mcl-1, including the estimation of their affinity for Mcl-1. Biological assays evidenced that the biological activity of Torsemide was lower as compared to the Deferasirox, which was able to efficiently and selectively inhibit the antiapoptotic activity of Mcl-1. Finally, docking and molecular dynamics led to a 3D model for the Deferasirox:Mcl-1 complex and revealed the positioning of the drug in the Mcl-1 P2/P3 pockets as well as almost all synthetic Mcl-1 inhibitors. Interestingly, contrary to known synthetic Mcl-1 inhibitors which interact through Arg263, Deferasirox, establishes a salt bridge with Lys234.

Conclusion: Deferasirox could be a potential candidate for drug repositioning as Mcl-1 inhibitor.

Keywords: drug repurposing, Mcl-1, Deferasirox, NMR, docking, dynamics

\section{Introduction}

Drug approval by the Food and Drug Administration (FDA) requires around 10 months, and the total drug development cost is estimated to be between $\$ 868$ million and \$1.241 billion USD. ${ }^{1}$ Advancing strategies to reduce drug development time, lower costs and improve success rates are essential. Drug repurposing is one such strategy. Also known as drug re-tasking or drug reprofiling, this approach describes the process that seeks to discover new indications for an existing drug that are not previously referenced and not currently investigated or prescribed..$^{2-5}$

The fact that drug repurposing is based on drugs with previous clinical approval has major benefits: data related to toxicology, preclinical safety, pharmacokinetics, and tolerance in healthy volunteers are already available for such compounds. A number of successes have been achieved in therapeutic areas such as sildenafil, an antihypertensive drug, for erectile dysfunction. ${ }^{3}$

Drug repurposing can be conveniently achieved using two strategies named activity-based drug repositioning and computational drug repositioning. The activity-based drug repositioning refers to the application of actual drugs for screening. In contrast, computational approaches are largely data-driven; they involve systematic analysis of public databases of any type (gene expression, chemical 


\section{Graphical Abstract}

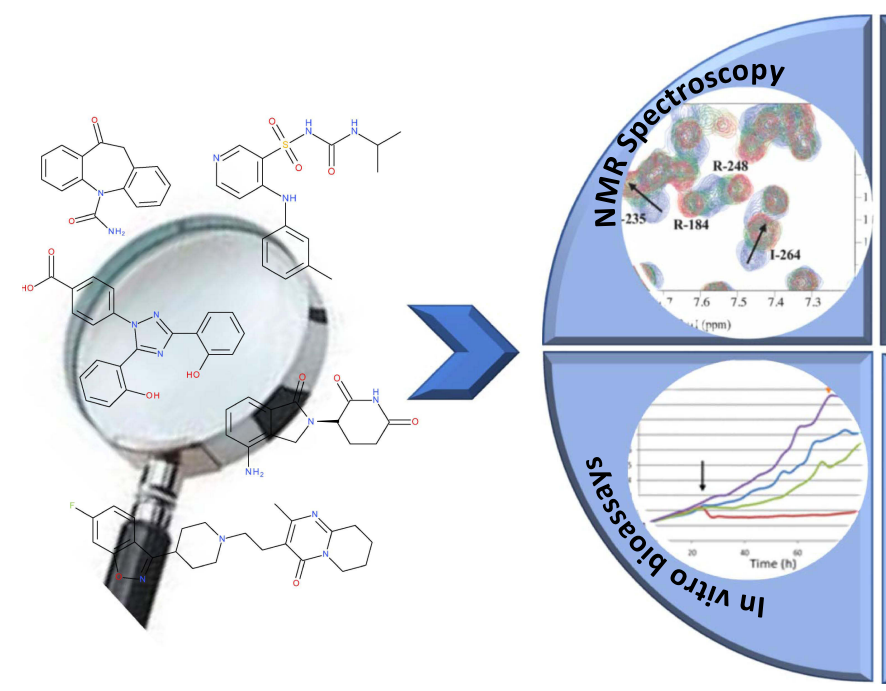

Experimental Validation

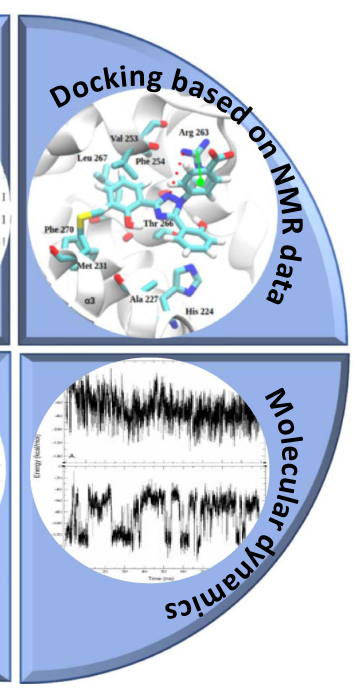

Binding mode determination

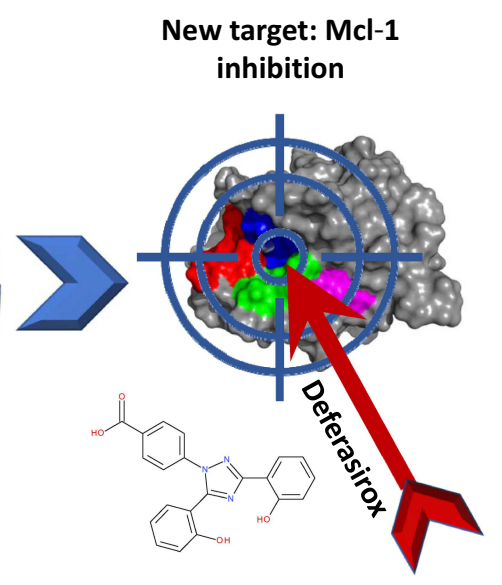

Drug repurposing for Cancer treatment structure ...) and use of bioinformatics tools, to systematically identify interaction networks between drugs and protein targets, which can then lead to the formulation of repurposing hypotheses. $^{5-8}$

In oncology, drug repurposing has drawn also particular attention and it has become now a powerful alternative for discovering and developing novel anticancer drug candidates. $^{7,9}$ The discovery of new anticancer drugs allows a rapid increasing of the drugs' number that can take part in combinatorial therapies.

In 2017, Glantz-Gashai et al published an in-silico drug repurposing study related to the oncology field. ${ }^{10}$ They have computationally screened a data set of all US Food and Drug Administration (FDA) approved drugs and a data set containing 2.3 million lead-like molecules randomly selected for the ZINC database against the antiapoptotic Myeloid Cell Leukemia-1 protein (Mcl-1). Mcl-1 is a pro-survival member of the $\mathrm{Bcl}-2$ protein family regulating the cell death. ${ }^{11,12}$ Mcl-1 is overexpressed in many human cancers. ${ }^{13}$ Restoring apoptosis and thus promoting tumor cell death is of significant therapeutic interest. Therefore, several small-molecules inhibiting Mcl-1 were developed during the last decade and are in clinical tests. ${ }^{14-23}$

Based on docking technique carried out on conformation ensembles, generated either by normal mode analysis (6 conformations), or by molecular dynamics (4 conformations) or from nuclear magnetic resonance data (20 conformations), Glantz-Gashai et al identified potential Mcl-1 ligands. ${ }^{10}$ From each screening, the top 10 ligands exhibiting the most favorable average binding energies were selected, resulting in 30 ligands in total. The 30 FDA-approved drugs predicted as potentially interacting with Mcl-1 were compared with their clinical use and half of them are already clinically used for cancer treatment by acting on other targets, thus promoting the idea that the other half of potential drugs could also be acting as anticancer agents. ${ }^{10}$ This in-silico study highlighted a set of FDA-approved drugs as potential Mcl-1 inhibitors and exposed them to the scientific community for further investigation (in vitro and in vivo activity evaluations).

In this context, we have focused on the validation of this theoretical study through experimental evidence. For this purpose, we selected five drugs from the 30 identified as potential inhibitors of $\mathrm{Mcl}-1$ protein, to prove and characterize their interaction with Mcl-1 protein, and to verify the possibility of repositioning them in cancer treatment. The choice of the five molecules (Oxcarbazepine, Deferasirox, Risperidone, Lenalidomide and Torsemide) (Table 1) was based mainly on (i) their predicted binding Gibbs energy, ${ }^{10}$ and (ii) their solubility in aqueous media according to the Drug Bank database. ${ }^{24}$ Interestingly, Deferasirox was selected in two screenings on FDA-approved drugs: the one carried out on normal mode ensemble as well as the one on molecular dynamics ensemble. 
Table I The FDA-Drugs Experimentally Evaluated in This Study

\begin{tabular}{|c|c|}
\hline FDA-Approved Drug ${ }^{a}$ & Predicted Binding Affinity to $\mathrm{Mcl}-\mathrm{I}^{\mathrm{b}}$ (kcal/mol) \\
\hline Oxcarbazepine & -5.99 \\
\hline Deferasirox & -7.53 or -6.03 \\
\hline Risperidone & -7.22 \\
\hline Lenalidomide & -6.23 \\
\hline Torsemide & -6.13 \\
\hline
\end{tabular}

Note: ${ }^{a}$ Data from Glantz-Gashai et al, 2017. ${ }^{10}$

Abbreviations: FDA, Food and Drug Administration; Mcl-I, myeloid cell leukemia-I.

Oxcarbazepine, a tricyclic compound with histaminergic and serotoninergic activity, is used to control pain, depression and as an antiemetic in cancer patients. Deferasirox is an oral iron chelator used to reduce chronic iron overload. ${ }^{25}$ Recently, Deferasirox was found to induce apoptosis of multiple myeloma cells via the inhibition of proline-rich tyrosine kinase 2 (Pyk2), which is known to promote tumor growth in multiple myeloma. ${ }^{26}$ Risperidone is an atypical antipsychotic with serotonin and dopaminergic activity. ${ }^{27}$ Lenalidomide is a structural analogue of the rhodamine derivative, BH3I, developed by Degterev et al. ${ }^{28}$ The BH3I molecule has a micromolar affinity for the BH3 domain of Mcl-1 protein. Lenalidomide is already associated with cancer treatment: it induces apoptosis and is used in the treatment of multiple myeloma and myelodysplastic syndromes. ${ }^{29,30}$ Finally, Torsemide, is a pyridine-sulfonylurea diuretic mainly used in the management of edema associated with congestive heart failure. ${ }^{31}$ It is interesting to note that Torsemide has some structural similarities with ABT-737, ${ }^{32}$ a promising drug inhibiting Bcl-2 and Bcl- $\mathrm{x}_{\mathrm{L}}$ another two anti-apoptotic proteins of Bcl-2 family, particularly through the presence of benzyl-sulfonyl-amide moiety.

Nuclear magnetic resonance (NMR), a powerful tool to characterize protein-ligand interactions in solution under near physiological conditions, ${ }^{33-35}$ was used to probe interactions at the atomic level between the five chosen drugs and Mcl-1. Biological assays were then carried out 
on the confirmed compounds from NMR study to demonstrate their in vitro action on the ovarian cancer cells. Molecular modelling study guided by NMR observations allowed us to predict the binding mode of Deferasirox and Torsemide. Finally, the most potent of the tested molecules according to the assays results is Deferasirox.

\section{Materials and Methods}

\section{Chemicals and Recombinant Mcl-I for NMR Studies}

Oxcarbazepine, Risperidone, Lenalidomide and Torsemide were purchased from Sigma-Aldrich and Deferasirox from VWR. Soluble labelled ${ }^{15} \mathrm{~N}$ Mcl-1 (172-327) was produced following a previously published protocol. ${ }^{36}$

\section{NMR Experiments}

The backbone assignment of Mcl-1 resonances was carried out in our previous work. ${ }^{36}$

To highlight the interaction and carry out the titration experiments between each drug and the labeled ${ }^{15} \mathrm{~N}$ Mcl-1, two sets of samples were prepared. The first set contained $600 \mu \mathrm{L}$ of a uniformly ${ }^{15} \mathrm{~N}$ labeled Mcl-1 solution prepared at $50 \mu \mathrm{M}$ in PBS buffer $(20 \mathrm{mM}, 130 \mathrm{mM} \mathrm{NaCl}, 2$ $\mathrm{mM}$ DTT, pH 7, 5\% $\left.\mathrm{D}_{2} \mathrm{O}, \mathrm{v} / \mathrm{v}\right)$. The second set contained ${ }^{15} \mathrm{~N}$ labeled Mcl-1 and drugs at Ligand/Protein $\left(\mathrm{L} / \mathrm{P}_{0}\right)$ ratio of 20/1 in PBS buffer (20 mM, $130 \mathrm{mM} \mathrm{NaCl,} 2 \mathrm{mM}$ DTT, $\mathrm{pH} 7,5 \% \mathrm{D}_{2} \mathrm{O}, \mathrm{v} / \mathrm{v}$ ) at a concentration of $50 \mu \mathrm{M}$ for protein and $1 \mathrm{mM}$ for drugs.

For Torsemide and Deferasirox, the titration series were incremented in steps of 1 or 2 molar equivalents of the ligand. To do this, adequate volumes of each sample were mixed to obtain the following ratios $\mathrm{L} / \mathrm{P}_{0}(0 / 1-1 / 1-$ $2 / 1-3 / 1-4 / 1-6 / 1-8 / 1-10 / 1-12 / 1-14 / 1-16 / 1-$ $17 / 1-18 / 1-19 / 1-20 / 1)$.

NMR spectra were recorded at $298 \mathrm{~K}$ on a Bruker Avance III $600 \mathrm{MHz}$ spectrometer equipped with a triple resonance cryoprobe $\left\{{ }^{1} \mathrm{H},{ }^{13} \mathrm{C},{ }^{15} \mathrm{~N}\right\}$ including shielded z-gradients.

The interaction between ${ }^{15} \mathrm{~N} \mathrm{Mcl}-1$ and drugs was characterized by recording $2 \mathrm{D}{ }^{1} \mathrm{H}^{-}{ }^{15} \mathrm{~N}$ HSQC experiments ${ }^{37}$ for each ratio $\mathrm{L} / \mathrm{P}_{0}$ described above.

2D ${ }^{1} \mathrm{H}^{15}{ }^{15} \mathrm{HSQC}$ spectra were collected with 128 indirect points, 1152 direct points using spectral width of $40 \mathrm{ppm}\left(\mathrm{F} 2,{ }^{15} \mathrm{~N}\right)$ and $12 \mathrm{ppm}\left(\mathrm{F} 1,{ }^{1} \mathrm{H}\right)$, respectively, and 64 scans. A one-second relaxation delay was used, and quadrature detection in the indirect dimension was obtained with states-time proportional phase incrementation (TPPI) phase cycling. ${ }^{38}$ Before Fourier transformation, linear prediction (LP) was applied in the indirect dimension (Forward LP on a complex data mode, Number of LP coefficients, and Number of points for LP were set to 64 and 32, respectively). The time domain data were zero filled (size of the real spectrum was $2048 \times 512$ points) and apodized by Sine Squared function. Data were acquired and processed using Topspin 3.5 software (Bruker Biospin).

Protein spectra were analyzed using Sparky for signal assignment, ${ }^{39} \mathrm{CCPNMR}^{40}$ and TITAN $^{41}$ for the estimation of $\mathrm{K}_{\mathrm{D}}$ and $\mathrm{k}_{\mathrm{off}}$.

For a given ${ }^{1} \mathrm{H}-{ }^{15} \mathrm{~N}$ HSQC cross peak, proton and nitrogen chemical shift perturbation induced by drug binding was defined as the combined difference between the corresponding chemical shifts in the bound and in the free states. The combined chemical shift perturbations (CCSP) were calculated from the ${ }^{1} \mathrm{H}$ and ${ }^{15} \mathrm{~N}$ shift coordinates of the cross peaks using Equation (1):

$$
\operatorname{CCSP}(\mathrm{ppm})=\left[\Delta \delta \mathrm{H}^{2}+(0.2 \Delta \delta \mathrm{N})^{2}\right]^{1 / 2}
$$

where $\Delta \delta \mathrm{H}$ and $\Delta \delta \mathrm{N}$ are the observed chemical shift differences along the proton and nitrogen dimensions, respectively, with respect to the $\mathrm{Mcl}-1$ free ${ }^{1} \mathrm{H}-{ }^{15} \mathrm{~N}$ HSQC spectrum.

The dissociation constant $\left(\mathrm{K}_{\mathrm{D}}\right)$ was then obtained by monitoring the chemical shift changes of the backbone amide as a function of ligand concentration using a binding model with $1: 1$ stoichiometry $^{42}$ in CCPNMR software ${ }^{40}$ (Equation 2).

$$
\Delta \delta_{\mathrm{obs}}=\frac{\Delta \delta_{\max }}{2}\left[1+\mathrm{x}+\frac{\mathrm{K}_{\mathrm{D}}}{\mathrm{P}_{0}}-\sqrt{\left(1+\mathrm{x}+\frac{\mathrm{K}_{\mathrm{D}}}{\mathrm{P}_{0}}\right)^{2}-4 \mathrm{x}}\right]
$$

where $\Delta \delta_{\text {obs }}$ is the change in the observed shift from the free state and fitted $\Delta \delta_{\max }$ is the maximum change in chemical shifts for the given peak, $x$ is the molar $\mathrm{L} / \mathrm{P}_{0}$ ratio and $\mathrm{P}_{0}$ is the total concentration of the labelled protein.

${ }^{1} \mathrm{H}-{ }^{15} \mathrm{~N}$ HSQC spectra were also analyzed by the TITAN program ${ }^{41}$ using a two-state binding mode. Mcl-1 concentration, drugs concentrations and NMR acquisition parameters were provided as inputs for the data fitting. Ten peaks with significant CCSPs were chosen to be fitted for each titration experiment. Error analysis was performed by 
the bootstrap re-sampling module in the program with 100 re-sampled spectra.

\section{Biological Assays}

\section{Cell Culture and Treatment}

IGROV1-R10 cells were obtained as previously described from IGROV1 cell line, ${ }^{43}$ itself kindly provided by Dr. Jean Bénard (Institut Gustave Roussy, Villejuif, France). This cell line was cultured in RPMI 1640 medium supplemented with $2 \mathrm{mM}$ GlutamaxTM, $25 \mathrm{mM}$ HEPES, 10\% fetal calf serum, and $33 \mathrm{mM}$ sodium bicarbonate (Fisher Scientific, Illkirch, France). The atmosphere was maintained at $37^{\circ} \mathrm{C}$ and $5 \% \mathrm{CO}_{2} .550,000$ cells were seeded in $25 \mathrm{~cm}^{2}$ flasks. After $24 \mathrm{~h}$, exponentially growing cells were exposed to Deferasirox or Torsemide $(0.1$ to $50 \mu \mathrm{M})$ as single agents or in combination with ABT-737 $(5 \mu \mathrm{M})$ or S63845 (1 $\mu \mathrm{M})$ for $48 \mathrm{~h}$.

\section{Real Time Cell Imaging}

Six thousand cells were seeded in 96-well plates in appropriate media. Cells were maintained at $37^{\circ} \mathrm{C}$ and $5 \% \mathrm{CO}_{2}$ and monitored using an IncuCyte ${ }^{\circledR} \mathrm{S} 3$ (Essen BioScience) and baseline images were taken using 10x objective. Phase-contrast images were acquired in real time every 2 hours from a single region per well. The live-cell phase contrast images were used to calculate confluence using the IncuCyte $^{\circledR}$ software, and to provide morphology information.

\section{Morphological Observation of the Cell Nuclei}

Both detached and adherent cells were pooled after trypsinization, applied to a polylysine-coated glass slide by cytocentrifugation and fixed with a solution of ethanol/ chloroform/acetic acid (6:3:1) after treatment. Preparations were then incubated for $15 \mathrm{~min}$ at room temperature with $1 \mu \mathrm{g} / \mathrm{mL}$ DAPI solution (Boehringer Mannheim-Roche, Mannheim, Germany), washed in distilled water, mounted under a coverslip in Mowiol (Calbiochem) and analyzed under a fluorescence microscope (BX51, Olympus, Rungis, France).

\section{Target Expression and Apoptosis Induction Analysis by} Western Blot

Cells were rinsed with ice-cold PBS, suspended in a lysis buffer [RIPA: $\mathrm{NaCl} 150 \mathrm{mM}$, Tris (pH 8) $50 \mathrm{mM}$, Triton X100 1\%, PMSF $4 \mathrm{mM}$, EDTA $5 \mathrm{mM}$, NaF $10 \mathrm{mM}$, $\mathrm{NaPPi} 10 \mathrm{mM}, \mathrm{Na}_{3} \mathrm{VO}_{4} 1 \mathrm{mM}$, aprotinin $0.5 \mu \mathrm{L} / \mathrm{mL}$ and $4.6 \mathrm{~mL}$ ultra-pure water] and incubated on ice for $30 \mathrm{~min}$.
Lysates were collected after centrifugation $(13200 \mathrm{~g}, 10$ min, $4^{\circ} \mathrm{C}$ ) and protein concentrations were determined using the Bradford assay (Bio-Rad, Hercules, USA). Equal amounts of protein $(25 \mu \mathrm{g})$ were separated by SDS-PAGE on a $4-15 \%$ gradient polyacrylamide MiniPROTEAN $^{\circledR}$ TGX $^{\mathrm{TM}}$ precast gel (Bio-Rad) and transferred to PDVF membranes (Bio-Rad). Membranes were blocked 1 hour at room temperature with $5 \%(\mathrm{v} / \mathrm{v})$ non-fat dry milk in TBS with $0.05 \%(\mathrm{v} / \mathrm{v})$ Tween 20 (T-TBS). Membranes were then incubated overnight at $4^{\circ} \mathrm{C}$ with the appropriate antibodies, listed above. Membranes were then washed with T-TBS and incubated for 1 hour with the appropriate secondary antibody. Signals were revealed using Enhance ChemiLuminescence substrate (ECL) Prime Western Blot detection reagent (GE Healthcare Life Sciences) and the ImageQuant $^{\circledR}$ Las4000Series (GE Healthcare Life Sciences).

Antibodies used: anti-Caspase 3 (\#9662), anti-PARP (\#9542), (Cell Signaling Technology), anti-Actin (\#MAB1501) (Merck Millipore).

\section{Apoptotic Events Quantification Using Flow Cytometry}

Adherent and floating cells were pooled by trypsinisation, washed with phosphate-buffered saline (PBS) and fixed with ethanol $70 \%$. Cells were then centrifuged at $2000 \mathrm{r}$. p.m. for $5 \mathrm{~min}$ and incubated for $30 \mathrm{~min}$ at $37^{\circ} \mathrm{C}$ in PBS, to allow the release of low molecular weight DNA. Cell pellets were stained with propidium iodide using the DNA Prep Coulter Reagent Kit (Beckman Coulter). Samples were analyzed using Gallios flow cytometer (Beckman Coulter) and cell cycle distribution was determined using Gallios software (Beckman-Coulter).

\section{FPA Experiments}

Carboxy-fluorescein labeled peptide (5-FAMEDIIRNIARHLAQVGDSMDR-NH2) and Bim-WT peptide (H-Ahx-DMRPEIWIAQELRRIGDEFNAYYAR-OH) were purchased from GENEPEP and used without further purification. Mcl-1 (172-327) was produced following a previously published protocol. ${ }^{36}$ FPA measurements were carried out in 96-well, black, flat-bottom plates (Greiner Bio-One) using the Biotek microplate reader Synergy 2. All assays were conducted in assay buffer containing 20 $\mathrm{mM} \mathrm{Na} \mathrm{HPO}_{4}(\mathrm{pH} 7.4), 50 \mathrm{mM} \mathrm{NaCl}, 2 \mu \mathrm{M}$ EDTA, $0.05 \%$ Pluronic F- 68 . For $\mathrm{IC}_{50}$ determination, compounds were diluted in DMSO in a 8-point, serial dilution scheme, added to assay plates and were incubated with $100 \mathrm{nM}$ Mcl-1 for two hours. Then, to measure inhibition of Mcl- 
1/FAM-Bid interaction, $15 \mathrm{nM}$ 5-FAM-Bid peptide was added and plates were incubated for two hours at room temperature. For each experiment, a negative control containing Mcl-1 and 5-FAM-Bid peptide (equivalent to $0 \%$ inhibition), and a positive control containing Mcl-1, 5FAM-Bid peptide, and $10 \mu \mathrm{M}$ Bim-WT peptide (equivalent to $100 \%$ inhibition) were included on each assay plate. Each point was duplicated and each experiment was performed in two biological replicates. The change in polarization was measured and used to calculate an $\mathrm{IC}_{50}$ (inhibitor concentration at which $50 \%$ of bound peptide is displaced), by fitting the inhibition data using GraphPad Prism 6 software to a sigmoidal, $4 \mathrm{PL}, \mathrm{X}$ is $\log$ (concentration). This was converted into a binding dissociation constant $\left(\mathrm{K}_{\mathrm{i}}\right)$ according to the formula described by Nikolovska-Coleska et al. ${ }^{44}$

\section{Docking and Molecular Dynamics Simulations Docking}

Mcl-1 X-ray structure (PDB ID 3WIX), ${ }^{45}$ with the ligand removed, was prepared using Schrödinger's Protein Preparation Wizard (PrepWizard) ${ }^{46}$ and used for the molecular docking. The Schrödinger's Protein Preparation Wizard generated tautomer with hydrogen on $\mathrm{N} \varepsilon_{2}$ nitrogen for His224 and His277 and tautomer with hydrogen on $\mathrm{N} \delta_{1}$ nitrogen for His 252 and His320. The Deferasirox and Torsemide starting X-ray structures were fetched from the Cambridge Structural Database (CCDC N $\mathrm{N}^{\circ} 872554$ and CCDC $\left.\mathrm{N}^{\circ} 795036\right) .{ }^{47-49}$ The protonation state of Deferasirox and Torsemide at $\mathrm{pH}=7$ was checked, and a deprotonated carboxylic group form was used in our modelling study of Deferasirox.

Docking was performed using the EA Dock DSS docking software ${ }^{50}$ through the swissDock server. ${ }^{51}$ In a nutshell, the binding modes are generated on all found binding cavities, and the CHARMM22 force-field is used to compute the binding energies. Then, the best interacting modes are post-processed using the implicit solvation model FACTS (Fast Analytical Continuum Treatment of Solvation $)^{52}$ to include the desolvation effect of ligand binding. Finally, the clustering of the binding modes is performed using a 2 radius, with a maximum of 8 binding modes per cluster.

Generated binding mode clusters from docking study were analyzed visually and two poses were retained depending on the orientation of the
Deferasirox and Torsemide in the binding groove, and their interaction with Mcl-1's main hotspots observed in the NMR study.

\section{Molecular Dynamics Simulations and Noncovalent Interactions Analysis}

The stability of the two selected binding poses for each ligand was then assessed using unbiased molecular dynamics simulations of 100 ns. All simulations were performed using NAMD 2.12 software. ${ }^{53}$ The all-atom CHARMM $36 \mathrm{~m}$ forcefield ${ }^{54,55}$ was applied for the protein and CGENFF $^{56}$ for ligands. The CHARMM-GUI server ${ }^{57}$ was used to prepare the starting systems. Each system was solvated using the TIP3P explicit water model ${ }^{58}$ within a rectangular box; the box size ensured that the simulated complex was at a minimum distance of $10 \AA$ from the edge. $0.15 \mathrm{M}$ of $\mathrm{NaCl}$ was added to neutralize the total charge of the system. Periodic boundary conditions were applied to the systems using the IMAGE algorithm. Van der Waals interactions were truncated using a force switching function between 10 and $12 \AA$ and the Particle Mesh Ewald (PME) $)^{59}$ was used to calculate long-range electrostatic interactions. All bonds involving hydrogen atoms were restrained by the SHAKE algorithm.

The systems underwent energy minimization in 10,000 steps. Next, the minimized systems were heated to $300 \mathrm{~K}$ and the dynamics were temperature-equilibrated during 50 ps via heating reassignment under NVT conditions. Finally, the systems ran freely for $100 \mathrm{~ns}$ under NPT conditions with a 2 fs time step. Langevin dynamics with a damping coefficient of $1 \mathrm{ps}^{-1}$ were used to maintain the system temperature and the Nosé-Hoover Langevin piston was used to maintain a $1 \mathrm{~atm}$ pressure. Production trajectories were saved every $10 \mathrm{ps}$ and resulted in 10,000 frames. These trajectories were analyzed using $\mathrm{VMD}^{60}$ and the Pycontact software ${ }^{61}$ for noncovalent interactions.

In order to account for noncovalent interactions and their evolution during the trajectory, a geometrical approach was used. In the first place, the interatomic distance matrix between ligand and the protein atoms was computed. Then, a cutoff distance of 5 was applied to consider an interaction and a sigmoid function, $\mathrm{f}(\mathrm{d})$, scored the interaction along the distance as follows (equation 3$):^{61}$

$$
f(d)=\left\{\begin{array}{c}
\frac{1}{1+\exp (5.0(d-4.0))}, d \leq \text { cutoff } \\
0, \text { else }
\end{array}\right.
$$


The interaction score, resulting from this sigmoid function was afterward encoded to an intensity color gradient per time unit.

\section{Results \& Discussion NMR Analysis}

We used an NMR approach based on the observation of protein signals to probe ligand binding to Mcl-1, to define protein hotspots and to quantify the binding affinity of each of the five chosen drugs for Mcl-1 (estimation of dissociation constant values $\mathrm{K}_{\mathrm{D}}$ ).

For this purpose, we expressed and purified ${ }^{15} \mathrm{~N}$ labeled Mcl-1 and recorded 2D ${ }^{1} \mathrm{H}_{-}-{ }^{15} \mathrm{~N}$ HSQC experiments in the absence and presence of each of the 5 ligands. The NMR assignment of Mcl-1 backbone resonances in the free form was previously obtained from the analysis of ${ }^{1} \mathrm{H},{ }^{15} \mathrm{~N}$, and ${ }^{13} \mathrm{C}$ triple resonance experiments. ${ }^{36,62}$

As shown in Figure 1, the presence of 20 molar equivalents of Risperidone, Lenalidomide, or Oxcarbazepine did not result in any modification of the $2 \mathrm{D}{ }^{1} \mathrm{H}^{-15} \mathrm{~N}$ HSQC spectrum of the protein. Mcl-1 amide cross peaks showed neither significant chemical shift perturbations nor changes in intensities, reflecting the lack of interaction between these 3 compounds and the protein.

The discrepancy between the computational study previously performed by Glantz-Gashai et $\mathrm{al}^{10}$ and this experimental validation can be due to different aspects. It is known that the scoring functions employed during the docking studies have some weakness, leading to discrepancies between docking and in vitro screening accuracy. ${ }^{63}$ The widely used docking programs predict ligand positions in the target similar to these observed by X-ray crystallography or NMR, but their scoring function does not necessarily rank it as the best. In addition, in the AutoDockVina program employed by Glantz-Gashai et al, ${ }^{10}$ the binding site in the protein is predefined by a box and so the choice of its position as well as its size could influence the results because it forces the ligands to position inside it. Furthermore, the success of docking depends also on the

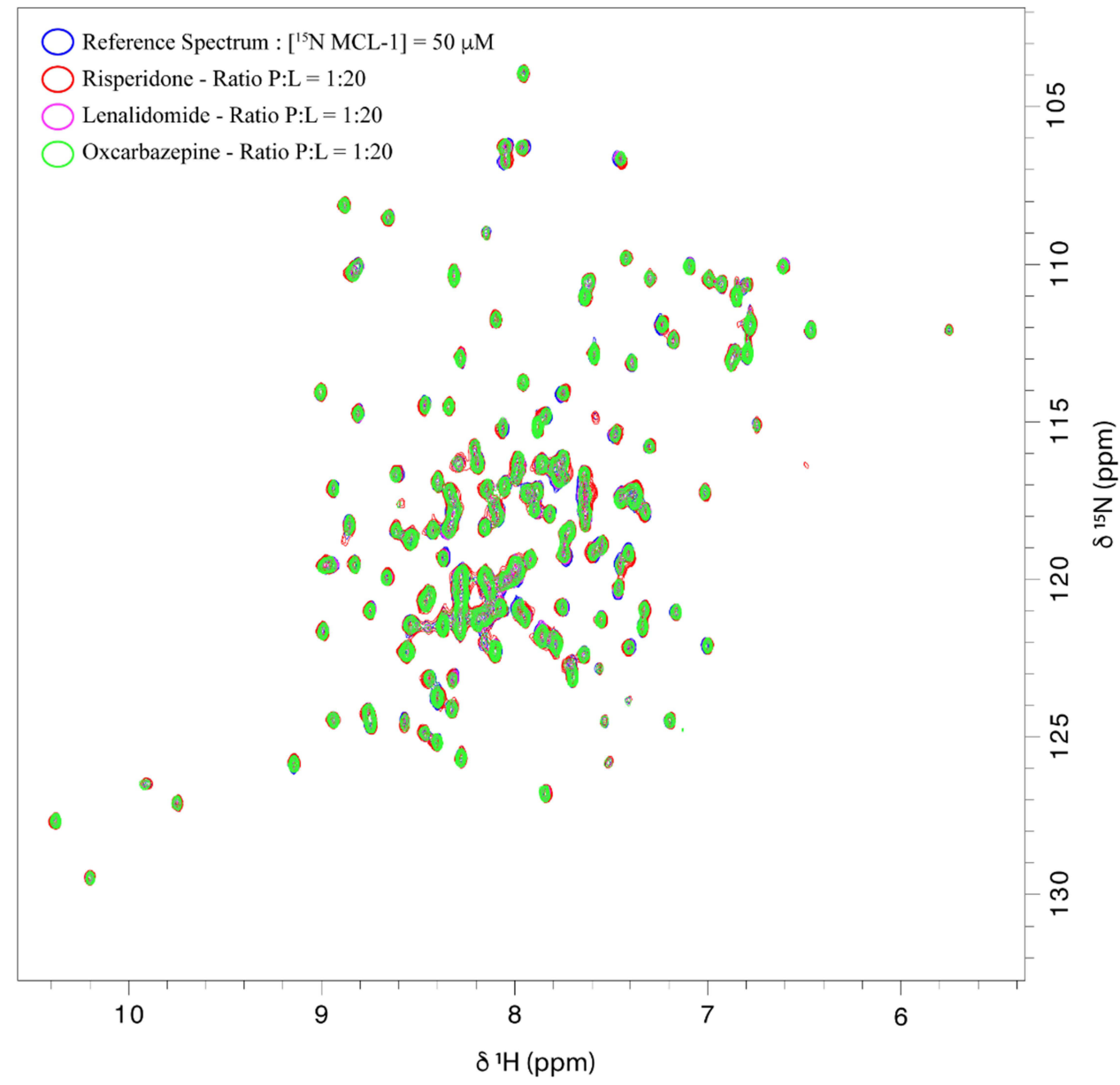

Figure I Overlay of ${ }^{1} \mathrm{H}_{-}{ }^{15} \mathrm{~N}$ HSQC spectra obtained for ${ }^{15} \mathrm{~N}-\mathrm{Mcl}-\mathrm{I}$ in the absence and presence of Risperidone, Lenalidomide or Oxcarbazepine. 
choice of protein structure conformation used in docking. For example, we previously demonstrated that Mcl-1 adopts an equilibrium between several conformations in its apo state but the opening of the P2 pocket, crucial for the synthetic ligands binding is only induced by the ligand presence. $^{64}$

The situation is different in the case of Torsemide and Deferasirox, which induce significant chemical shift perturbations (CSP) in the ${ }^{1} \mathrm{H}^{15}{ }^{15} \mathrm{NSQC}$ spectrum of the protein. We therefore focused on the characterization of the interaction between Mcl-1 and these two compounds separately.

\section{Mcl-I: Torsemide}

The comparison of ${ }^{1} \mathrm{H}-{ }^{15} \mathrm{~N}$ HSQC spectra recorded in the absence and presence of 20 molar equivalent of Torsemide (Figure 2) allowed us to calculate combined CSP values (CCSP) for each protein residue (Figure $3 \mathrm{~A}$ ) and to identify protein regions involved in the ligand binding.
As shown in Figure $3 \mathrm{~A}$ and $\mathrm{B}$, protein residues exhibiting the highest CCSP values are mostly localized in the canonical BH3 binding groove of Mcl-1 and include Leu267, Trp261, His252, Met250, Ile264, Leu235, Leu232, Phe270, Val253, Val216, Gly219, Glu221, Val220 and Arg263. These residues form a continuous surface on only one side of the protein and are mainly located at and around hydrophobic pockets P1, P2, and P4 of the Mcl-1 canonical binding site. ${ }^{36}$

Large CCSP values were also observed for residues Val243, Ser245, and Arg248 of helix $\alpha 4$ that are close to hotspot residues Met250, His252, and Val253 in the Mcl-1 structure (Figure 3C). The perturbation of Lys238 in the loop $\alpha 3-\alpha 4$ (Figure 3C) is likely due to a conformational rearrangement induced by its spatial proximity with the $\mathrm{P} 1$ hydrophobic pocket.

The contact surface formed by the perturbed residues is much larger compared to the size of the Torsemide, ie, in a 1:1 stoichiometry model, and the Torsemide could unlikely occupy simultaneously both hydrophobic pockets P2 and

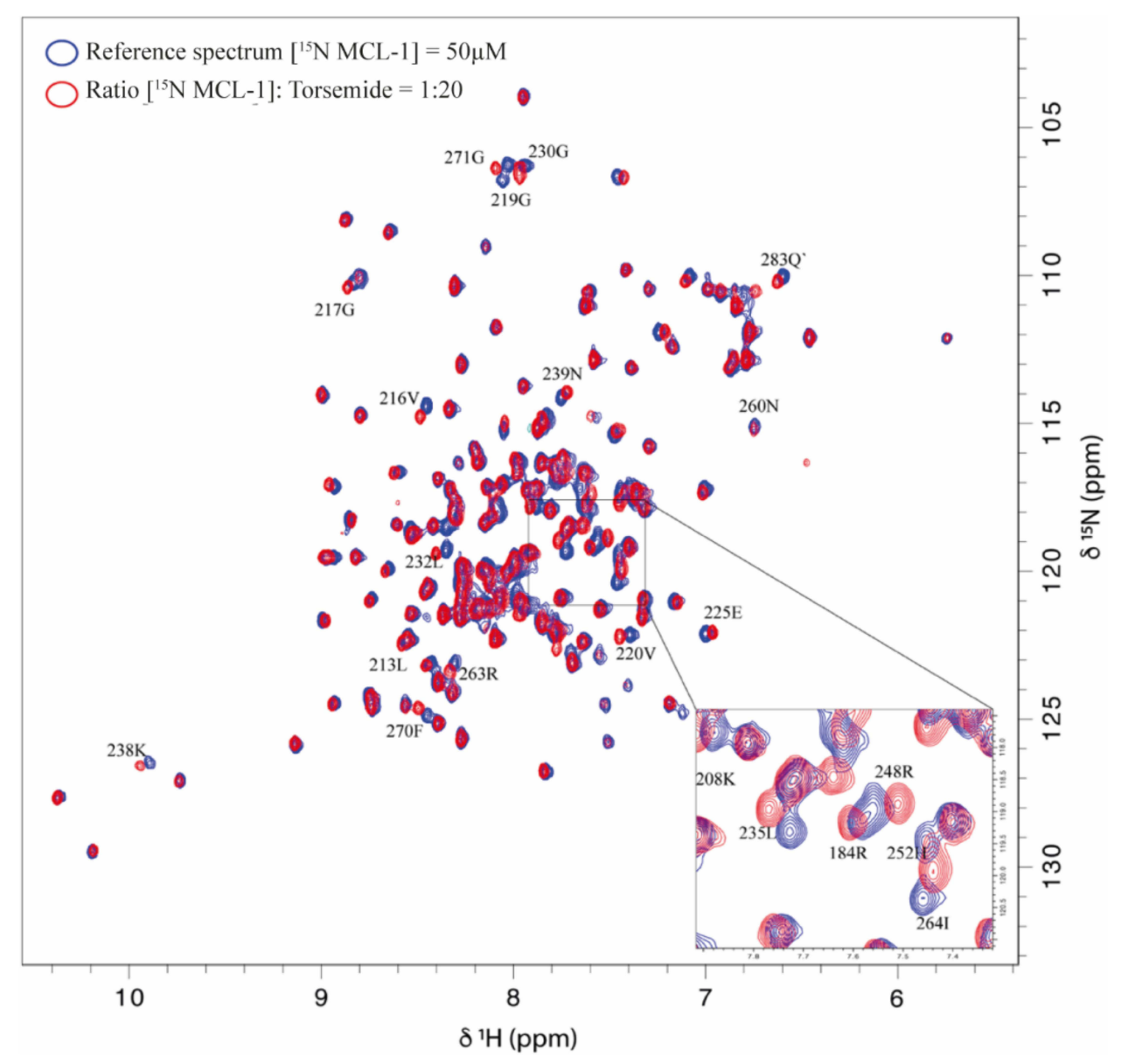

Figure 2 Overlay of ${ }^{1} \mathrm{H}-{ }^{15} \mathrm{~N}$ HSQC spectra obtained for ${ }^{15} \mathrm{~N}-\mathrm{Mcl}-\mathrm{I}$ in the absence and presence of Torsemide. 


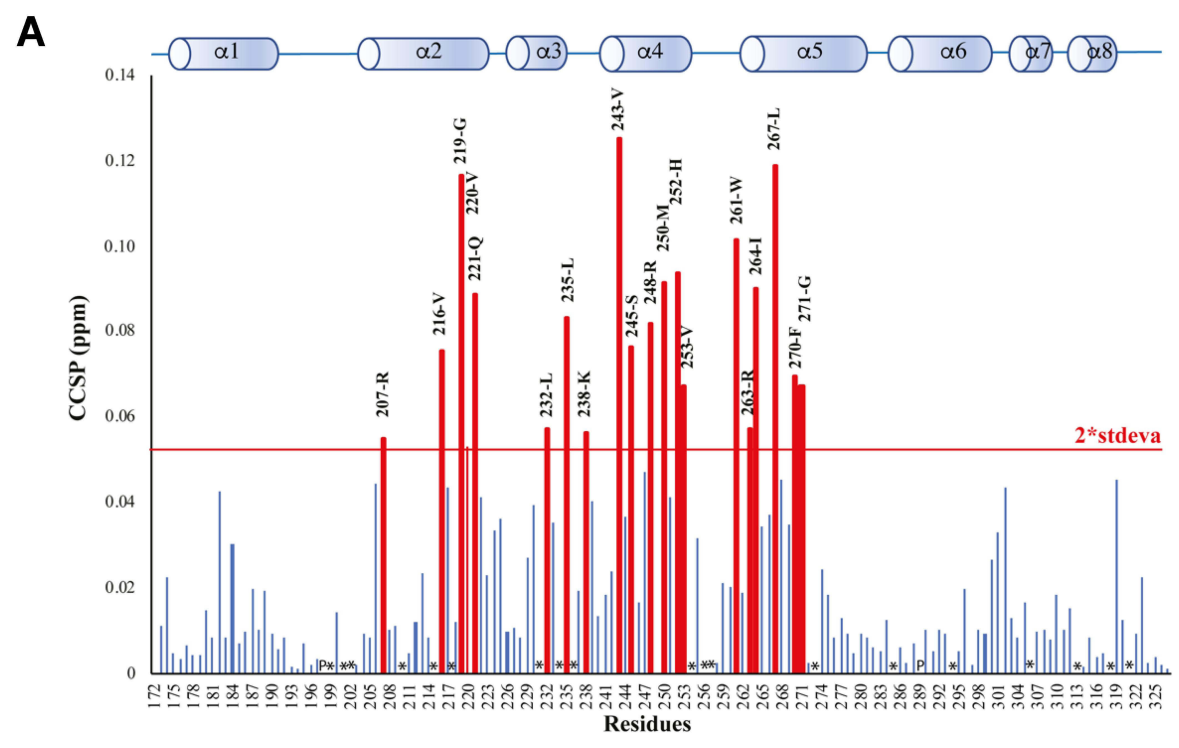

B

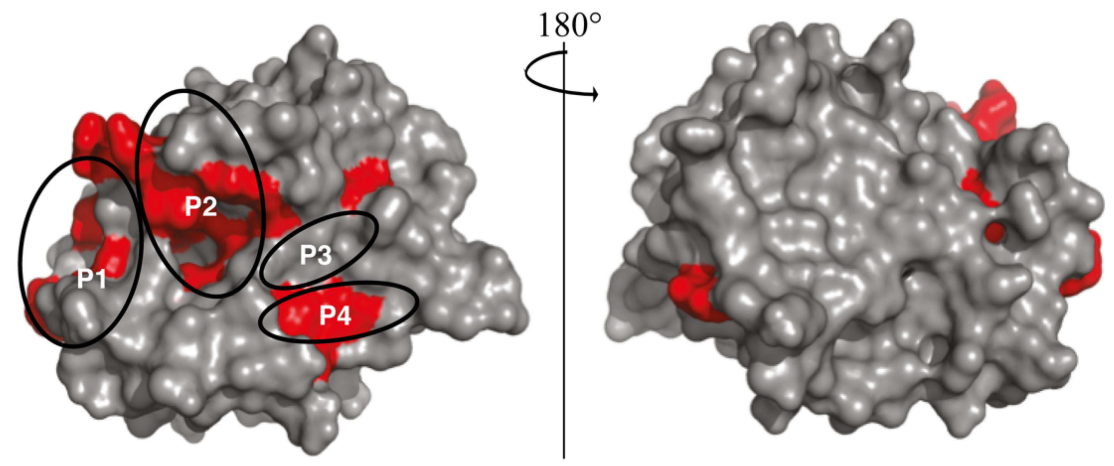

C
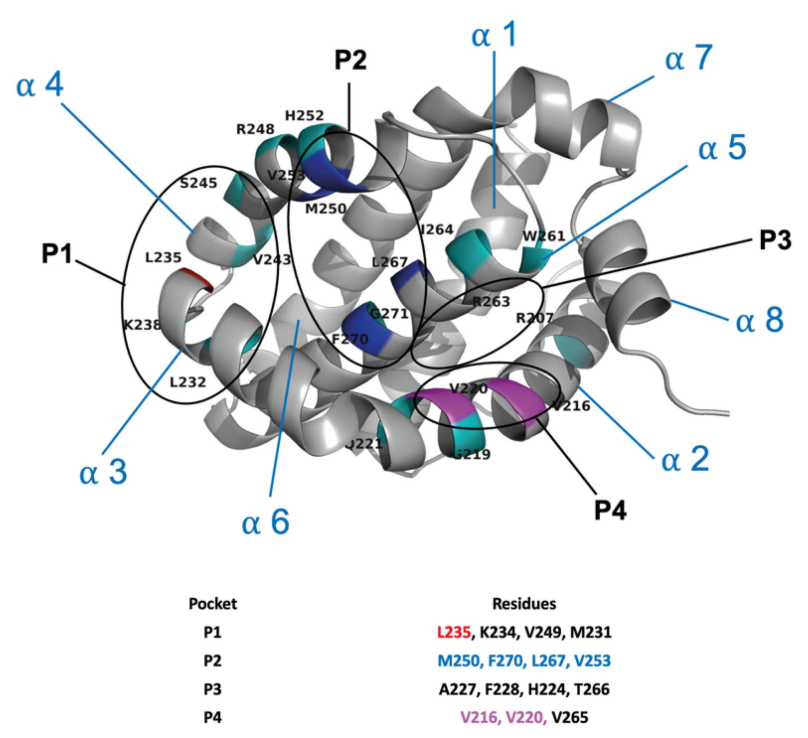

Other perturbed residues : R207, G219, Q221, L232, K238, V243, S245, R248, H252, W261, R263, I264, and G271

Figure 3 (A) Measured Combined Chemical Shifts Perturbation (CCSP) of the Mcl-I amide functions after the addition of Torsemide (ratio ${ }^{15} \mathrm{~N}-\mathrm{Mcl}-\mathrm{I}$ :Torsemide I:20). (P) presence of Proline. $\left({ }^{*}\right)$ Undetermined. (B) The most perturbed residues on the surface of the Mcl-I protein. (C) The cartoon representation of Mcl-I structure with annotation of most perturbed residues (red=residues from PI, Blue=residues from P2, Purple= residues from P4, Green=other residues). 
P4. Thus, the chemical shift changes of P4 residues Val216, Gly219, Val220, and Glu221 may result from a direct binding to Torsemide or from their spatial proximity with the P2 hydrophobic pocket.

NMR titration experiments were also conducted to quantify the binding affinity $\left(\mathrm{K}_{\mathrm{D}}\right)$ for Torsemide. NMR crosspeaks of the ${ }^{1} \mathrm{H}^{15} \mathrm{~N}$ HSQC spectrum showed linear concentrationdependent chemical shift changes upon addition of increasing amounts of the ligand (Figure 4A), indicating a fast exchange regime on the NMR chemical shift time scale. ${ }^{42}$ In this case, the evolution of the ${ }^{1} \mathrm{H}_{-}{ }^{15} \mathrm{~N}$ chemical shifts with the protein/

\section{A}

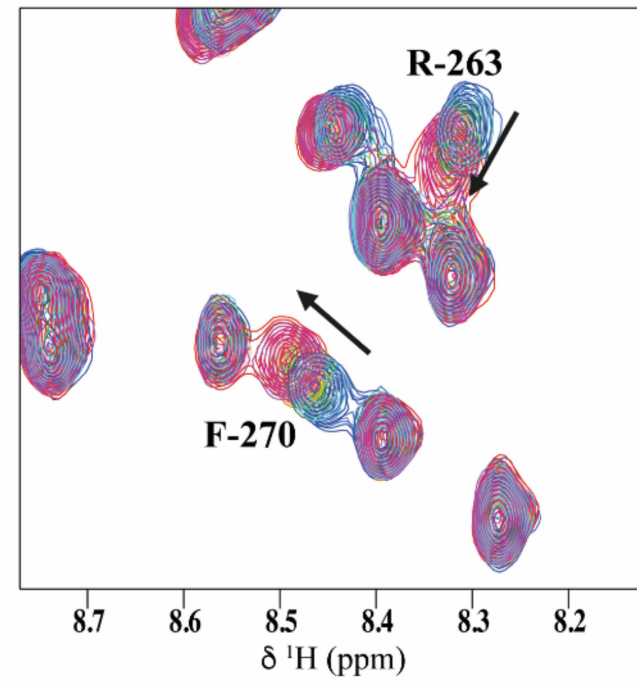

-122.5
-123.0
-123.5
-124.0
-124.5
-125.0
-125.5
-126.0

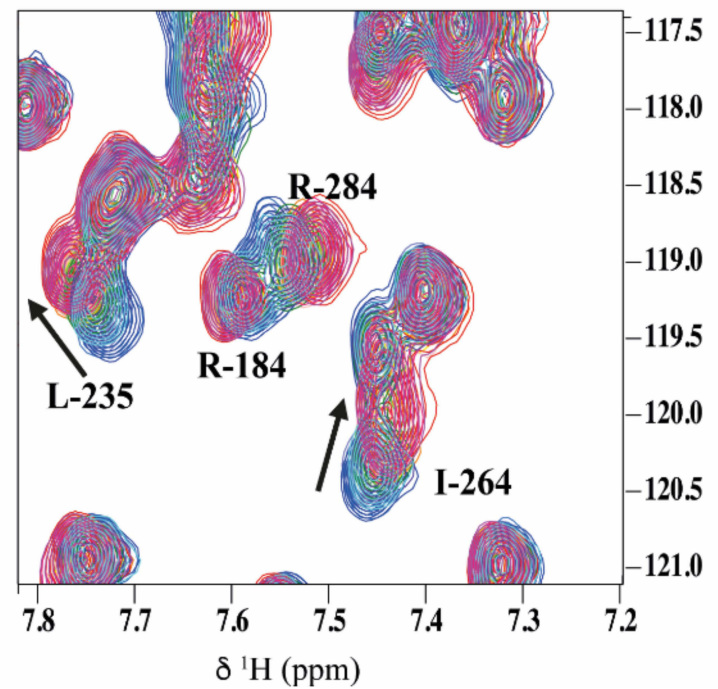

Torsemide molar ratio can be fitted to determine apparent thermodynamic affinities at the residue level (Figure 4B). Apparent $\mathrm{K}_{\mathrm{D}}$ for the most perturbed residues were estimated in the millimolar range, with an average value of $1 \mathrm{mM}$.

2D line-shape analyses of the NMR crosspeaks, carried out using the TITAN software, ${ }^{41}$ allowed the estimation of the kinetic $\left(\mathrm{k}_{\mathrm{off}}\right)$ and thermodynamic $\left(\mathrm{K}_{\mathrm{D}}\right)$ constants. Interestingly, it revealed a slow $\mathrm{k}_{\mathrm{off}}$ value $\left(818 \pm 8 \mathrm{~s}^{-1}\right)$ and a low residence time $\tau$ of $1.2 \mathrm{~ms}\left(\tau=1 / \mathrm{k}_{\text {off }}\right)$. These values are consistent with the low affinity of Torsemide for the Mcl-1 protein.

B
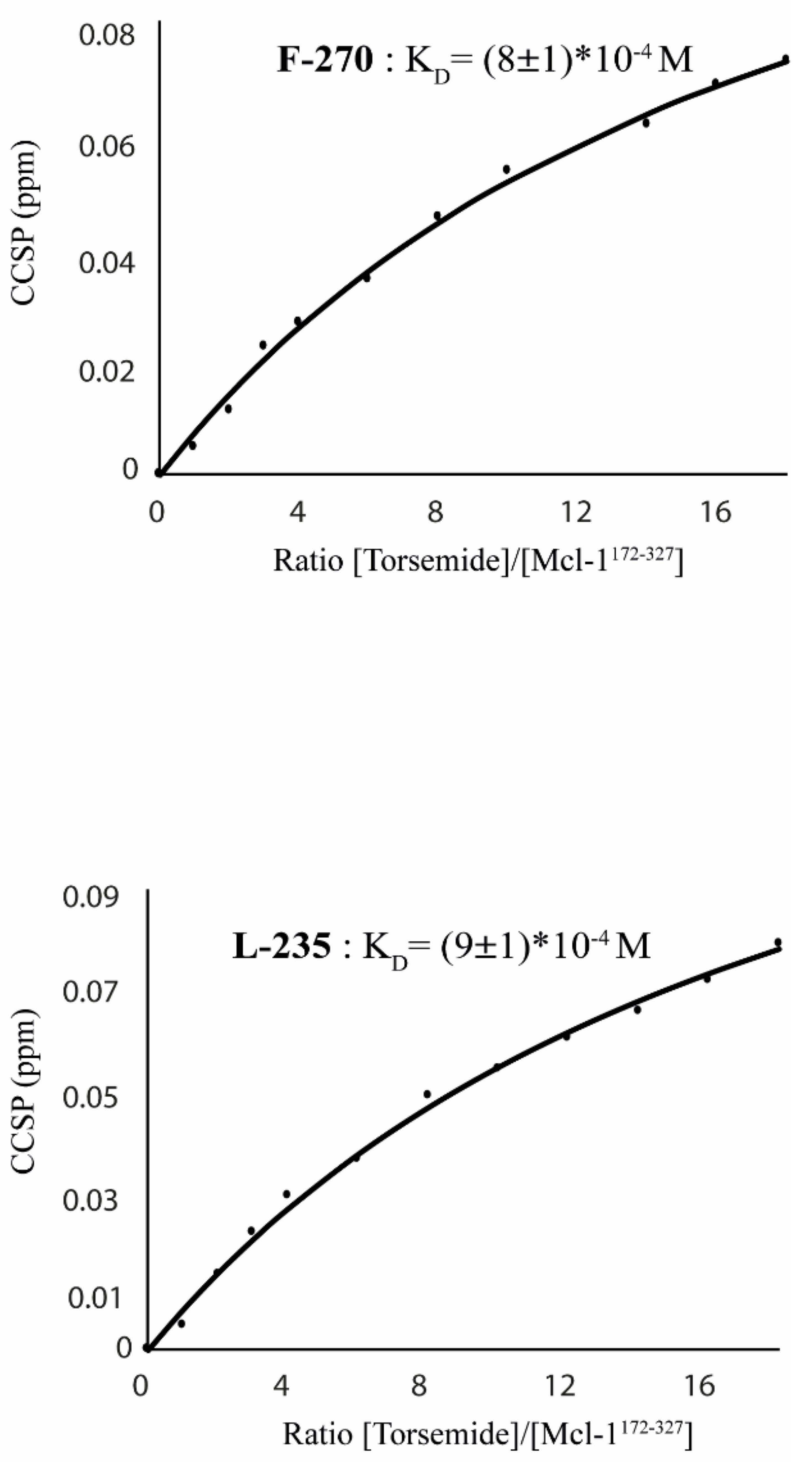

Figure 4 Overlay of ${ }^{~} \mathrm{H}_{-}{ }^{15} \mathrm{~N}$ HSQC spectra (expanded views) obtained for ${ }^{15} \mathrm{~N}-\mathrm{Mcl}-\mathrm{I}$ in the absence and presence of increasing amounts of Torsemide (various $\mathrm{L} / \mathrm{P}_{0}$ ratio). Chemical shift modifications of residues F-270, R-263, L-235, R-184, R-284 and I-264 (A), are indicated by black arrows. (B) Titration curves for F-270 and L-235 and K estimation of each residue using Equation (2). 


\section{Mcl-I: Deferasirox}

Using the same approach, the analysis of ${ }^{1} \mathrm{H}-{ }^{15} \mathrm{~N}$ HSQC spectra of Mcl-1 recorded in the absence and presence of increasing amounts of Deferasirox also revealed significant chemical shift perturbations (Figure 5). This observation reflects the presence of an interaction between this drug and Mcl-1. The highest CCSP values correspond to residues located either in the hydrophobic pockets (P1: Lys234, Leu235/ P2: Val253, Leu267, Phe270/ P3: Phe228/ P4: Val216, Val220) or in the areas surrounding them (Gly219, Arg263, Val243, Ile264, Asp256, Trp261, Asp236, Gly271, Arg248, Gly217, Lys238, Thr259 and Leu232) (Figure 6A and B).

As observed for Torsemide, the mapping of the residues, perturbed by Deferasirox on the surface of Mcl-1, reveals a continuous contact zone on the same side of the protein centered on pocket P2 (Figure 6B and $\mathrm{C}$ ). However, the observed CCSP values were higher for Deferasirox compared to Torsemide, suggesting that Deferasirox has a higher affinity for Mcl1 protein.

Accordingly, a dissociation constant $\left(\mathrm{K}_{\mathrm{D}}\right)$ of approximately $300 \mu \mathrm{M}$ was extracted from the fitting of CCSP values as a function of protein/ligand molar ratio, confirming that Mcl-1 shows a 3-fold greater affinity for Deferasirox than for Torsemide (Figure 7).

The $\mathrm{k}_{\mathrm{off}}, \mathrm{K}_{\mathrm{D}}$ and $\tau$ values of $368 \pm 8 \mathrm{~s}^{-1}, 262 \mu \mathrm{M}$ and $2.71 \mathrm{~ms}$, respectively, were also calculated using the TITAN software and reflect the higher affinity of Deferasirox for the Mcl-1 protein.

As for Torsemide and other previously studied ligands, it was observed that the identified contact surface is larger compared to the Deferasirox size (Figure 6C). This suggests that some NMR perturbations induced by Deferasirox could be related to an indirect effect of the interaction. $^{36}$

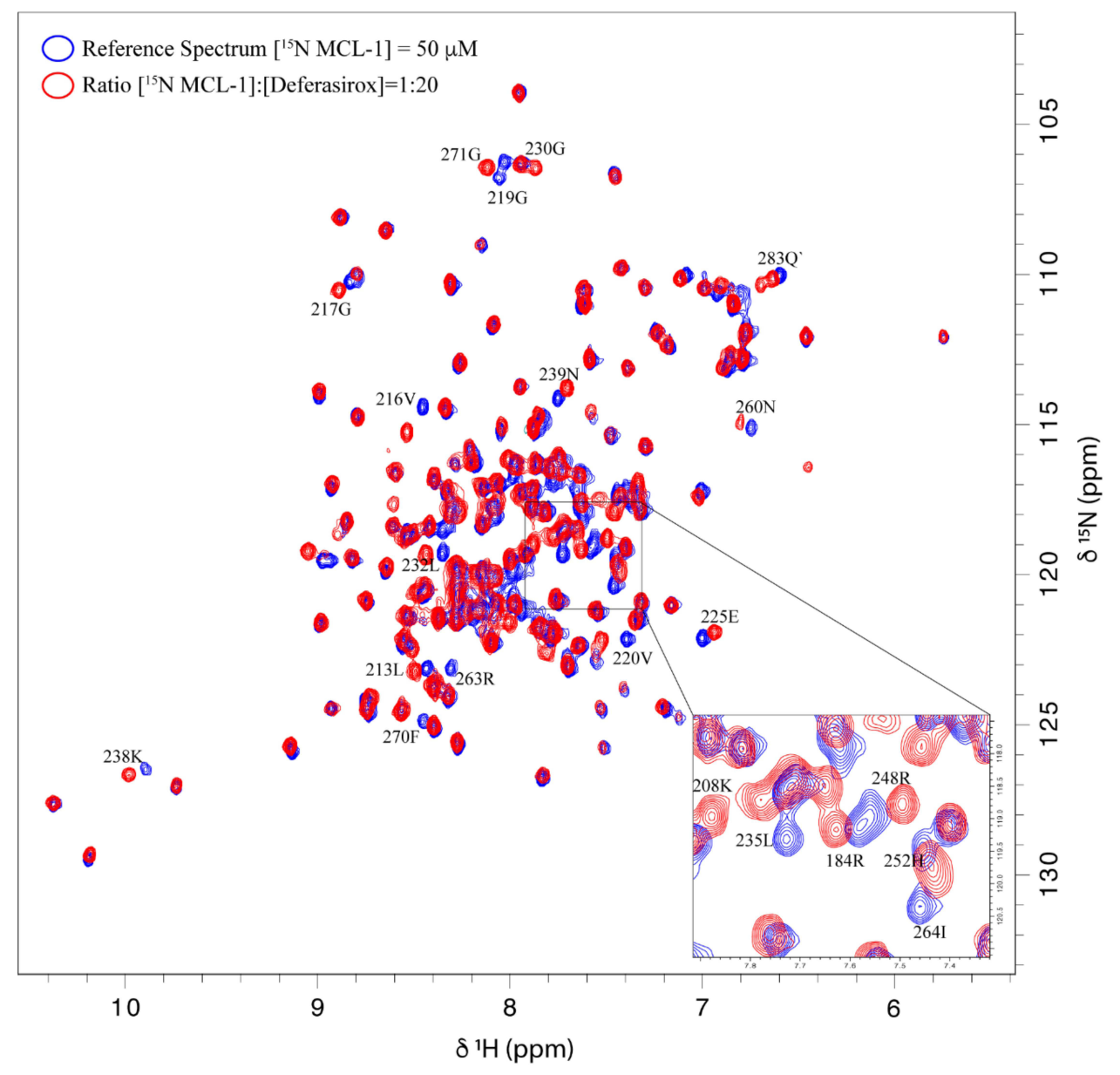

Figure 5 Overlay of ${ }^{1} \mathrm{H}_{-}{ }^{15} \mathrm{~N}$ HSQC spectra obtained for ${ }^{15} \mathrm{~N}-\mathrm{Mcl}-\mathrm{I}$ in the absence and presence of Deferasirox. 

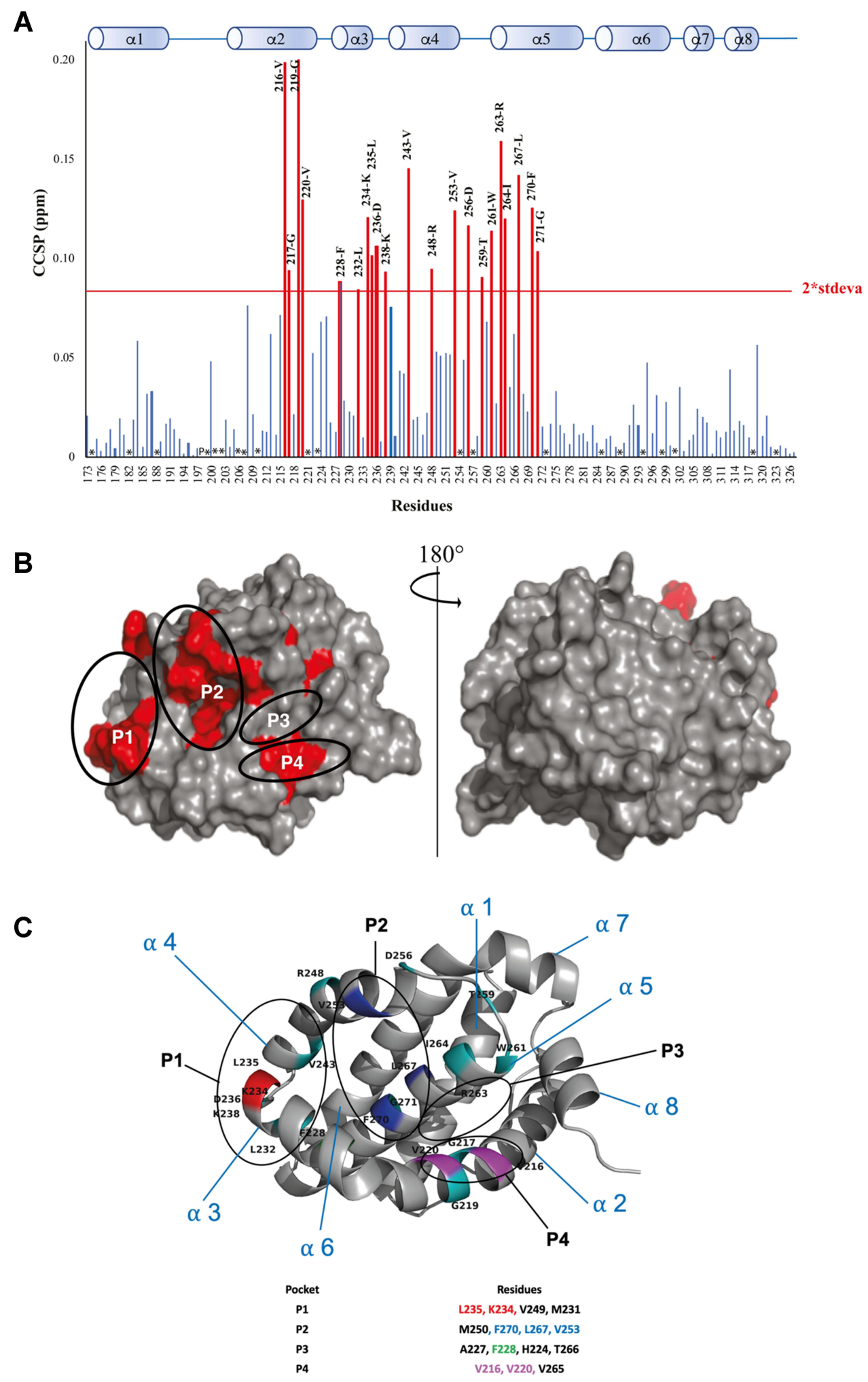

Other perturbed residues : G217, G219, L232, D236, K238, V243, R248, D256, T259, W261, R263, G271

Figure 6 (A) Measured Combined Chemical Shifts Perturbation (CCSP) of the Mcl-I amide functions after the addition of Deferasirox (ratio ${ }^{15} \mathrm{~N}-\mathrm{Mcl}-\mathrm{I}:$ Deferasirox I:20). (P) presence of Proline. $\left(^{*}\right)$ Undetermined. (B) The most perturbed residues on the surface of the Mcl-I protein. (C) The cartoon representation of Mcl-I structure with annotation of most perturbed residues (red=residues from PI, Blue=residues from P2, Purple= residues from P4, Green=other residues). 
A
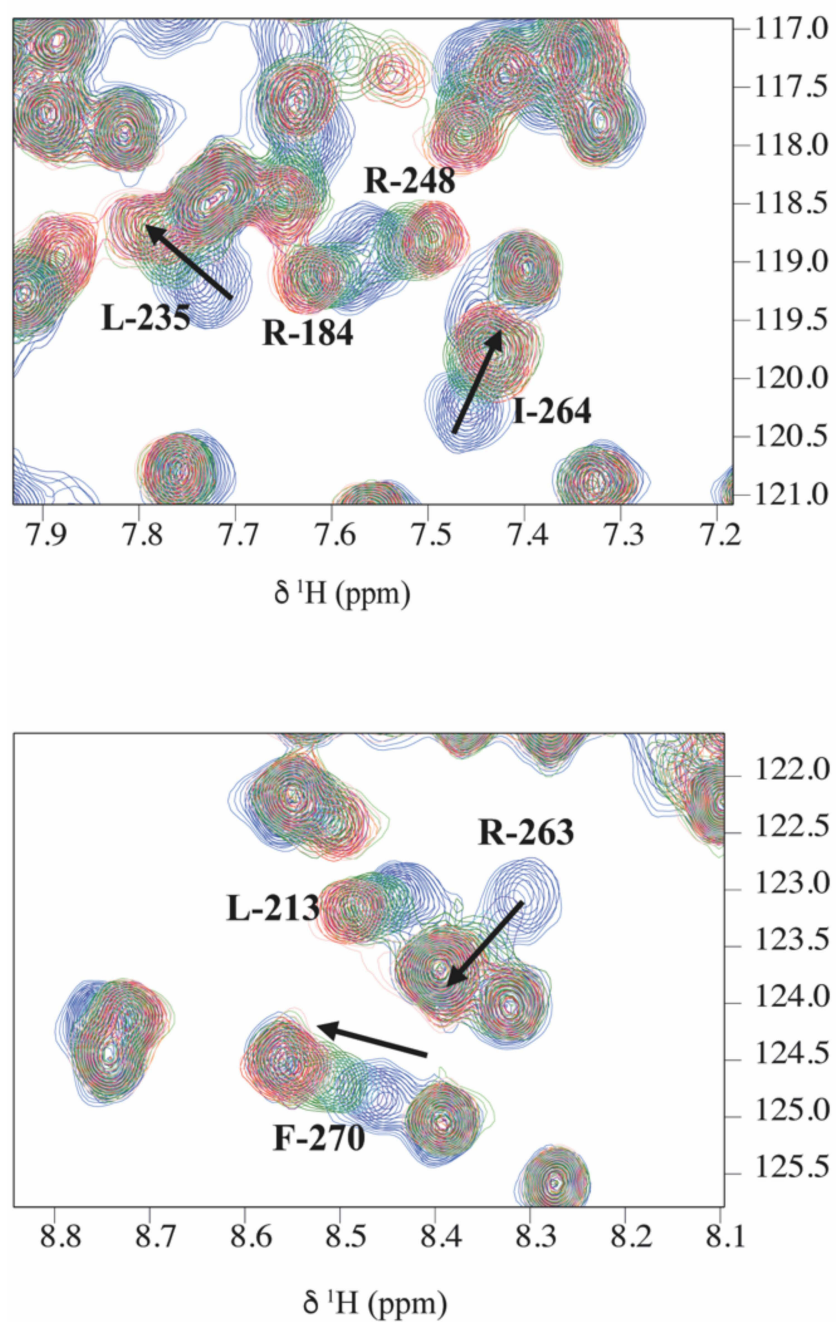

B

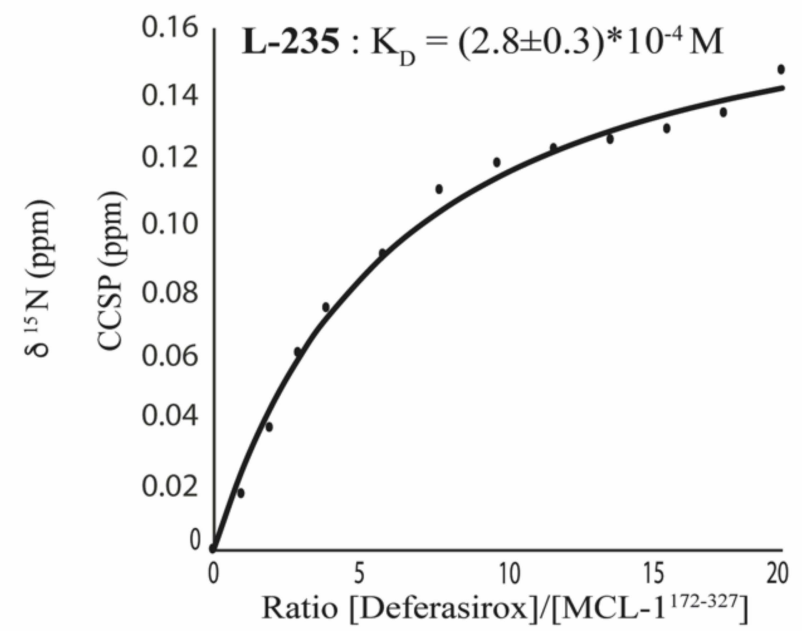

F-270 $: \mathrm{K}_{\mathrm{D}}=(4.3 \pm 0.4)^{*} 10^{-4} \mathrm{M}$

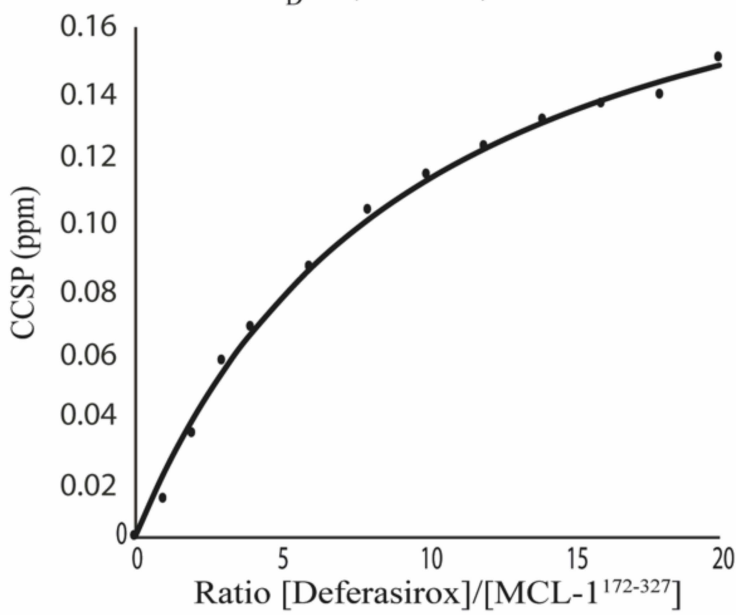

Figure 7 Overlay of ${ }^{1} \mathrm{H}-{ }^{15} \mathrm{~N}$ HSQC spectra (expanded views) obtained for ${ }^{15} \mathrm{~N}-\mathrm{Mcl}-\mathrm{I}$ in the absence and presence of increasing amounts of Deferasirox (various $\mathrm{L} / \mathrm{P}_{0}$ ratio). Chemical shift modifications of residues L-235, R-I84, R-248, I-264, L-2I3, R-263 and F-270 (A), are indicated by black arrows. (B) Titration curves for L-235 and F-270 and $K_{D}$ estimation of each residue using Equation 2.

\section{Biological Assay}

We next performed biological assays to validate the effect of Deferasirox and Torsemide on Mcl-1 anti-apoptotic activity. The chemoresistant IGROV1-R10 ovarian cancer cell line was used since it is known to be in a steady state for apoptosis entry following concomitant Mcl-1 and Bcl$\mathrm{x}_{\mathrm{L}}$ inhibition as previously described ${ }^{65} \mathrm{Mcl}-1$ inhibition by the molecules is assessed by a treatment of IGROV1R10 cells in combination with ABT-737, a well-known Bcl- $\mathrm{x}_{\mathrm{L}}$ inhibitor. In this context, Mcl-1 inhibitors induce massive apoptosis as shown by the effect of the combination of the selective Mcl-1 inhibitor S63845 with ABT-737 on IGROV1-R10 cells (Figure S1).
We first investigated the effect of both compounds from 0.1 to $50 \mu \mathrm{M}$ on IGROV1-R10 cells by real-time imaging in 96-well plates. Deferasirox did not induce cytotoxic effects as single agent even at $50 \mu \mathrm{M}$. In contrast, Torsemide at 50 $\mu \mathrm{M}$ decreases proliferation as single agent.

Deferasirox and Torsemide have then been tested in association with ABT-737 to confirm their potential activity and selectivity on Mcl-1 suggested by NMR studies.

For Deferasirox, $25 \mu \mathrm{M}$ appeared as the optimal concentration to obtain the best cytotoxic effect in combination with ABT-737 (Figure 8). A concentration-dependent slowed down proliferation was suggested by the reduced cell confluency. Moreover, cell detachment and cell 

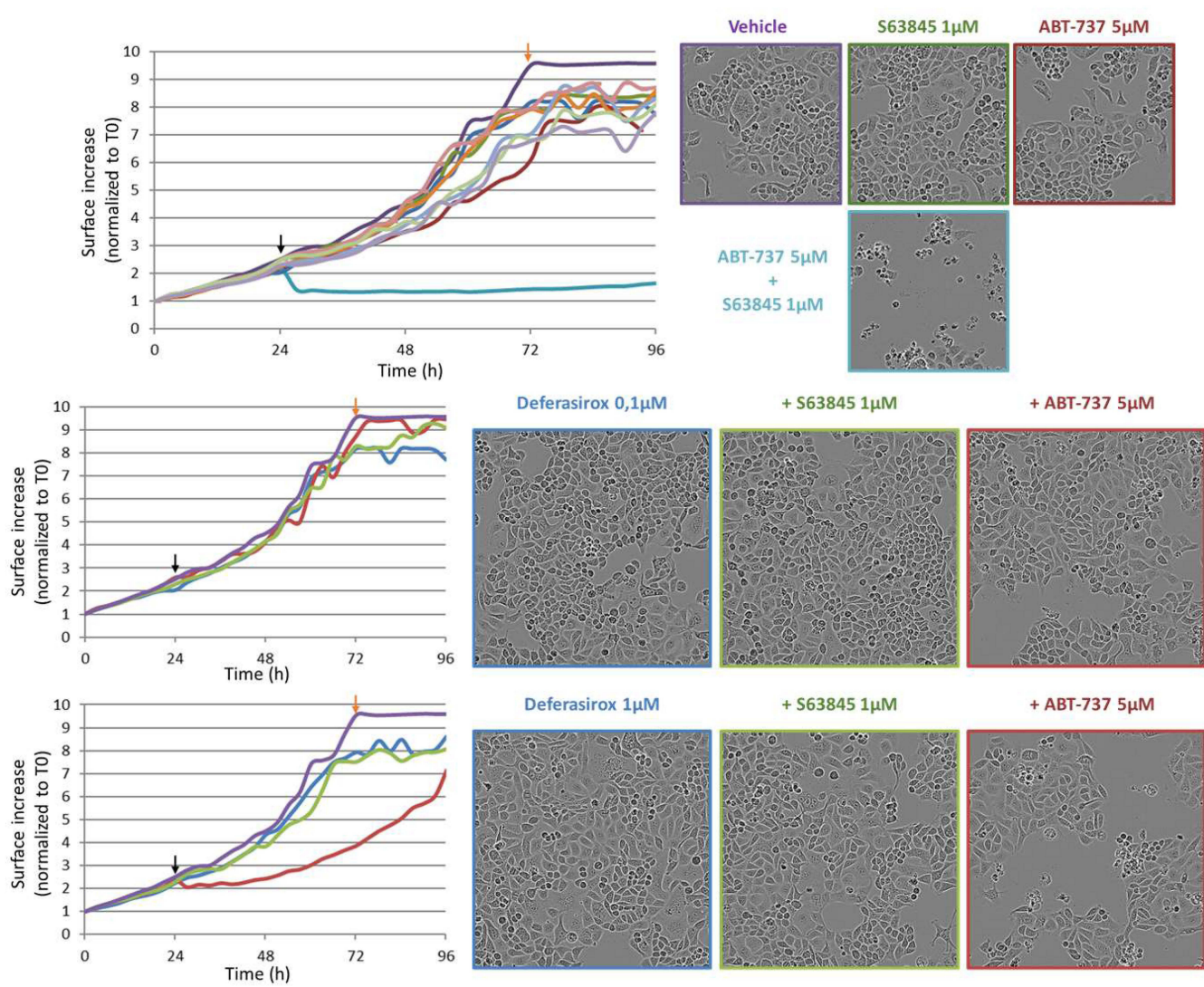

Deferasirox $1 \mu \mathrm{M}$

$+\mathrm{S} 638451 \mu \mathrm{M}$

+ ABT-737 $5 \mu \mathrm{M}$
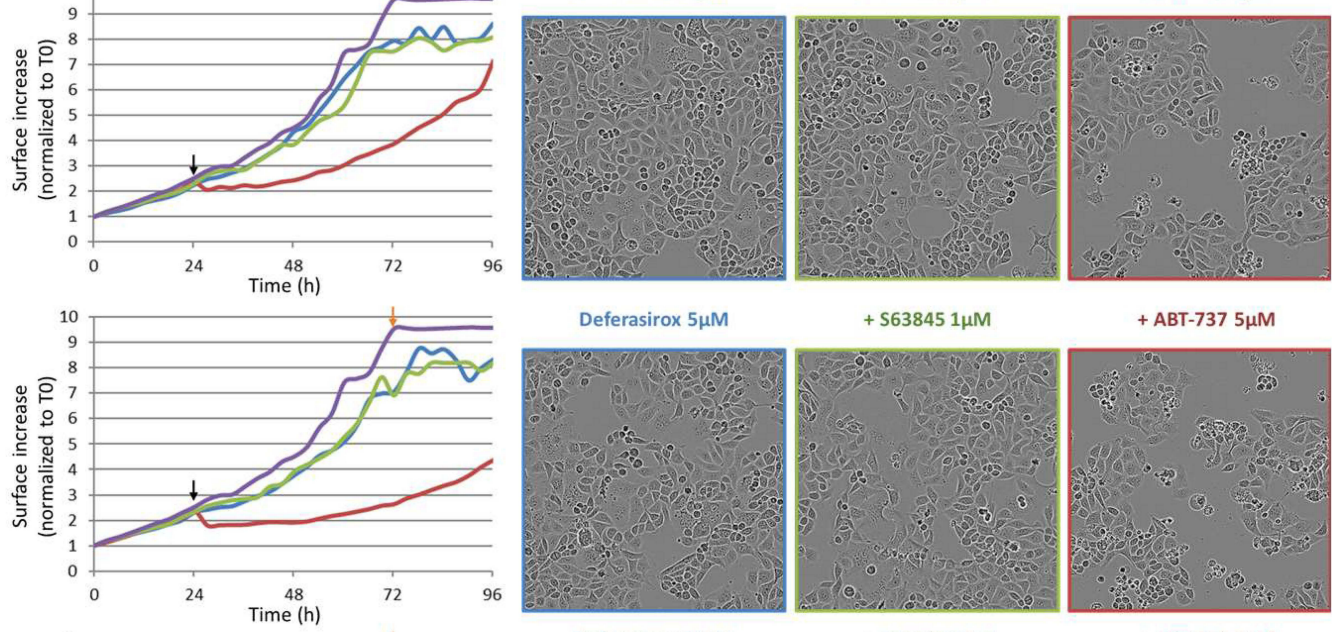

Deferasirox $5 \mu \mathrm{M}$

$+\mathrm{S} 638451 \mu \mathrm{M}$

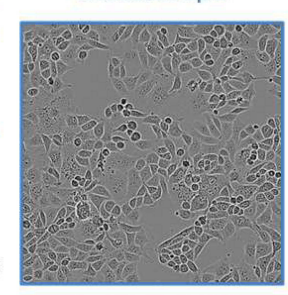

Deferasirox $25 \mu \mathrm{M}$

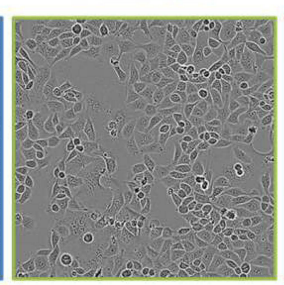

+ S63845 1 $\mu \mathrm{M}$
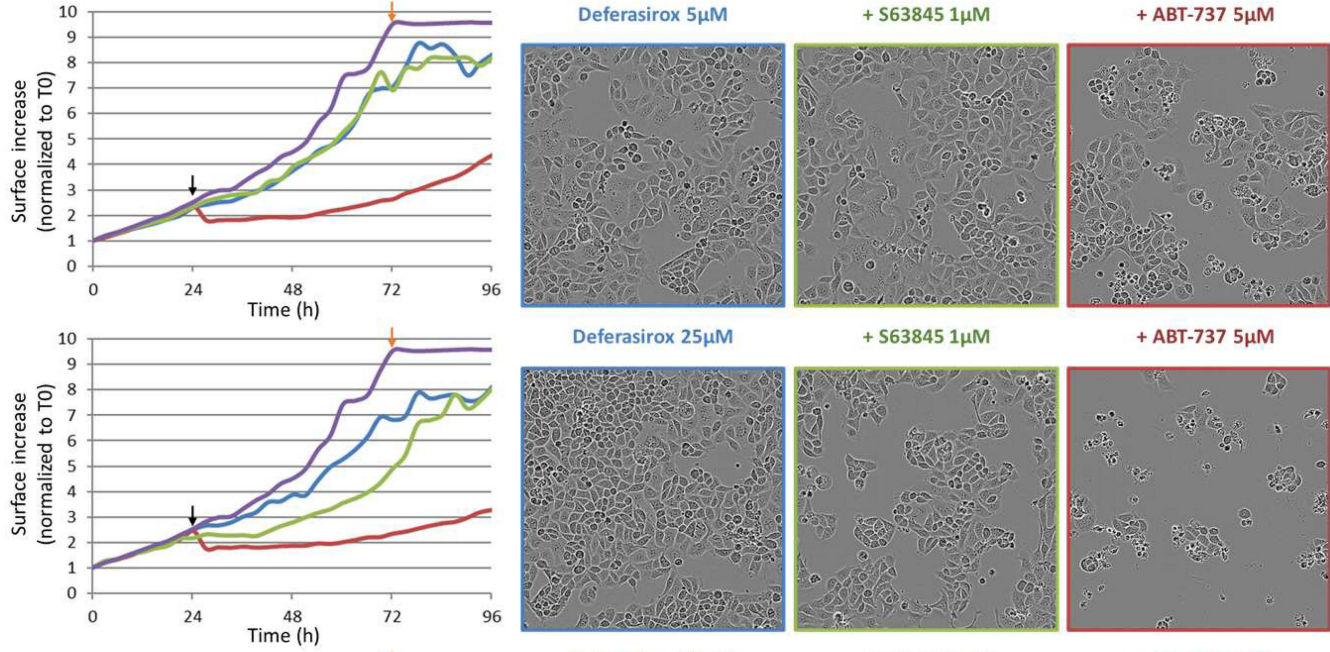

+ ABT-737 $5 \mu \mathrm{M}$

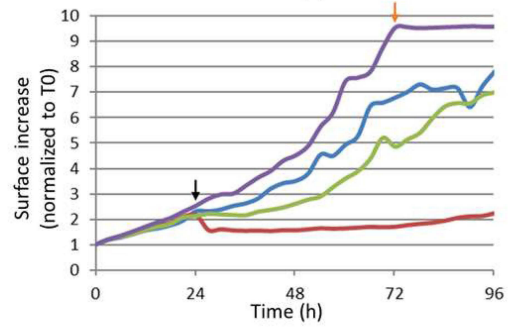

Deferasirox $50 \mu \mathrm{M}$

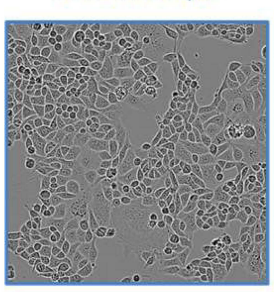

+ S63845 1 $\mu \mathrm{M}$

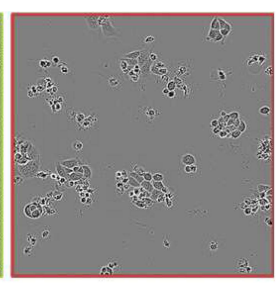

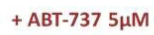
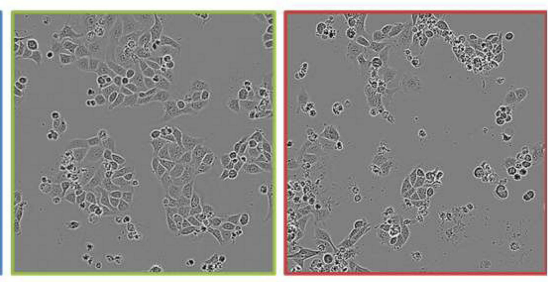

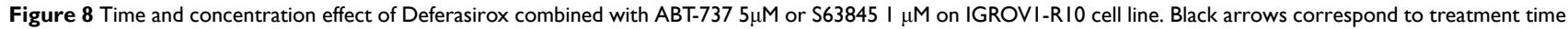
and Orange arrows highlight the $48 \mathrm{~h}$ exposure time, corresponding to photos. 
shrinking were observed when Deferasirox was combined to ABT-737.

The selective activity of the compounds on Mcl-1 as compared to Bcl- $\mathrm{x}_{\mathrm{L}}$, was also evaluated using a combination with S63845. Selectivity for Mcl-1 should result in a massive apoptosis induction in combination with ABT-737 but not with S63845. Conversely, if the molecule is able to bind to $\mathrm{Bcl}-\mathrm{x}_{\mathrm{L}}$ and inhibits its activity, cells will undergo apoptosis in combination with S63845. In our experiments, Deferasirox did not induce cytotoxic effect when combined to S63845, suggesting its specificity for Mcl-1 as compared to Bcl- $\mathrm{x}_{\mathrm{L}}$ (Figure 8).

In contrast, Torsemide appeared as a less potent Mcl-1 Inhibitor. Indeed, at $25 \mu \mathrm{M}$, only a weak cytotoxic effect is observed in combination with ABT-737. At $50 \mu \mathrm{M}$, Torsemide exerts a cytotoxic effect when combined to ABT-737, but also a marked cytostatic effect as a single agent (Figure 9).

In order to complete these results and to demonstrate the apoptotic nature of cell death, we observed cells and nuclear morphologies as well as DNA content histograms (cell cycle repartition). After a $48 \mathrm{~h}$ exposure, neither 25 $\mu \mathrm{M}$ Deferasirox nor $25 \mu \mathrm{M}$ Torsemide induced apoptosis as single agents (Figure 10). When they were combined to S63845, little or no modification of cells and nuclei morphologies or DNA content histograms were observed, strongly suggesting that the molecules are not able to inhibit Bcl- $\mathrm{x}_{\mathrm{L}}$, as expected. In contrast, when Deferasirox was combined to ABT-737, a great number of floating and shrunk cells was observable, suggesting a high level of cell death. The observation of nuclei morphologies after DAPI staining showed numerous nuclear condensations and fragmentations evocative of apoptotic cell death, as well as the appearance of a sub-G1 peak (Figure 10A). Lastly, Western blot revealed an intense PARP and Caspase 3 cleavages only in cells exposed to the Deferasirox/ABT737 combination, confirming the apoptotic nature of cell death (Figure 10). In contrast, the combination of Torsemide with either ABT-737 or S63845 did not exert more effect than the one observed with the molecules used separately.

Taken together, our results showed that Deferasirox is able to efficiently inhibit Mcl-1 in a dose and time-dependent manner with optimal conditions reached with $25 \mu \mathrm{M}$ and a $48 \mathrm{~h}$ exposure. The biological activity of Torsemide seems to be lower as compared to the one of Deferasirox since a $50 \mu \mathrm{M}$ concentration is required to observe similar effects, this concentration being itself associated to a cytostatic effect of Torsemide used as a single agent.

\section{Competition Binding Assays by FPA}

In order to determine if the mechanism of Deferasirox killing IGROV1-R10 in combination with ABT-737 is through inhibiting Mcl-1, and to see the effect of Deferasirox on disrupting Mcl-1 interactions with proapoptotic partners like Bid, competition binding assays using Fluorescence polarization anisotropy (FPA) were carried out. The results confirmed a direct interaction between Mcl-1 and Deferasirox and evidenced that Deferasirox was able to disrupt interactions between Mcl-1 with Bid. An inhibition close to $60 \%$ (at $600 \mu \mathrm{M}$ ) was observed and $\mathrm{IC}_{50}$ and $\mathrm{K}_{\mathrm{i}}$ values of $368 \pm 45 \mu \mathrm{M}$ and $83 \pm 10 \mu \mathrm{M}$, respectively, were obtained for Deferasirox (Figure S2).

Torsemide was also tested. As expected, a weaker inhibition close to $20 \%$ was observed at a concentration of $600 \mu \mathrm{M}$. This inhibition was too weak to allow the estimation of $\mathrm{IC}_{50}$ and $\mathrm{K}_{\mathrm{i}}$ values.

\section{Deferasirox and Torsemide Binding Modes Through Molecular Modeling}

To elucidate, in detail, the binding modes of Torsemide and Deferasirox in Mcl-1, a docking study followed by molecular dynamics simulation was carried out. Three X-ray structures of Deferasirox ${ }^{47,66,67}$ and Torsemide $^{49}$ are available in the Cambridge Structural Database. The three structures of Deferasirox are closely related and share a common intramolecular H-bond between one hydroxyl group and the nitrogen atom of the triazole group. We docked the Deferasirox X-ray structure (CCDC N 872554) and Torsemide one (CCDC $\mathrm{N}^{\circ}$ 795036) to the Mcl-1 model (PDB ID: 3WIX) using swissDock server. We applied the blind docking approach during which ligands binding poses in the vicinity of all Mcl-1 cavities were generated. For Deferasirox, among 29 clusters resulting from docking, only one was elsewhere than in the canonical binding site (Figure S3A). While for Torsemide, a total of 56 clusters were generated and 4 clusters were outside of the canonical binding site (Figure S3B). None of the Torsemide clusters nor Deferasirox ones occupied the $\mathrm{P} 4$ pocket. We retained two binding poses of Deferasirox and two of Torsemide from the docking results, based on the perturbed residues revealed by the NMR study. In the case of Deferasirox, the 

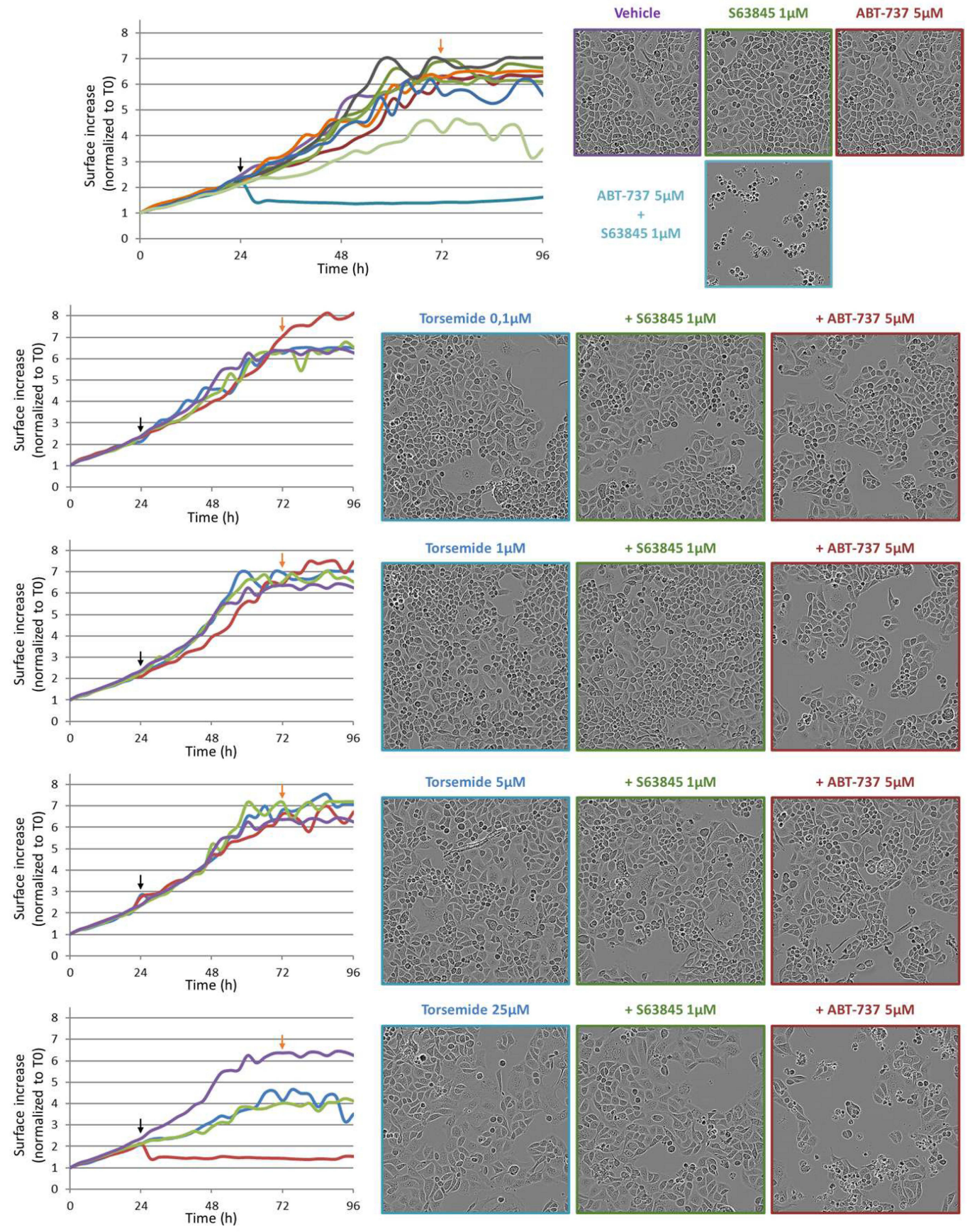

\section{+ ABT-737 5 $\mu \mathrm{M}$}
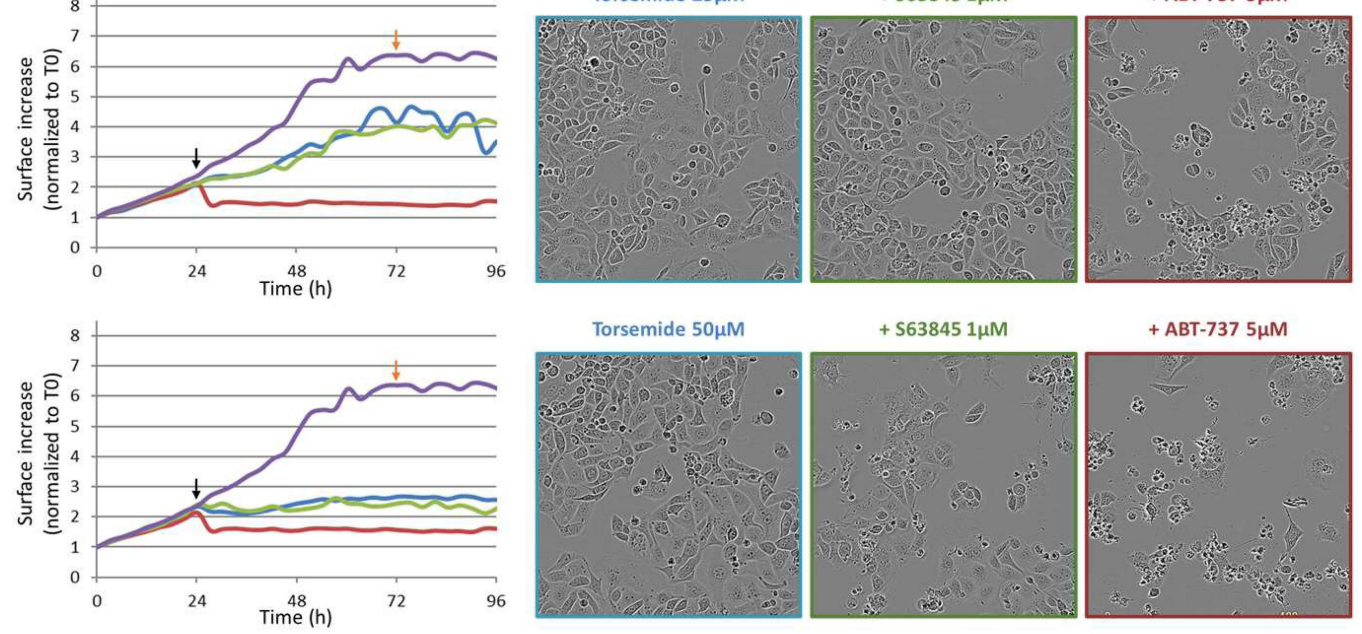

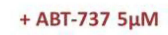

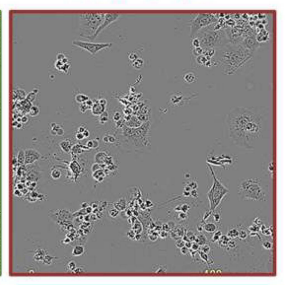

Figure 9 Time and concentration effect of Torsemide combined with ABT-737 $5 \mu \mathrm{M}$ or S63845 I $\mu \mathrm{M}$ on IGROVI-RI0 cell line. Black arrows correspond to treatment time and Orange arrows highlight the $48 \mathrm{~h}$ exposure time, corresponding to photos. 


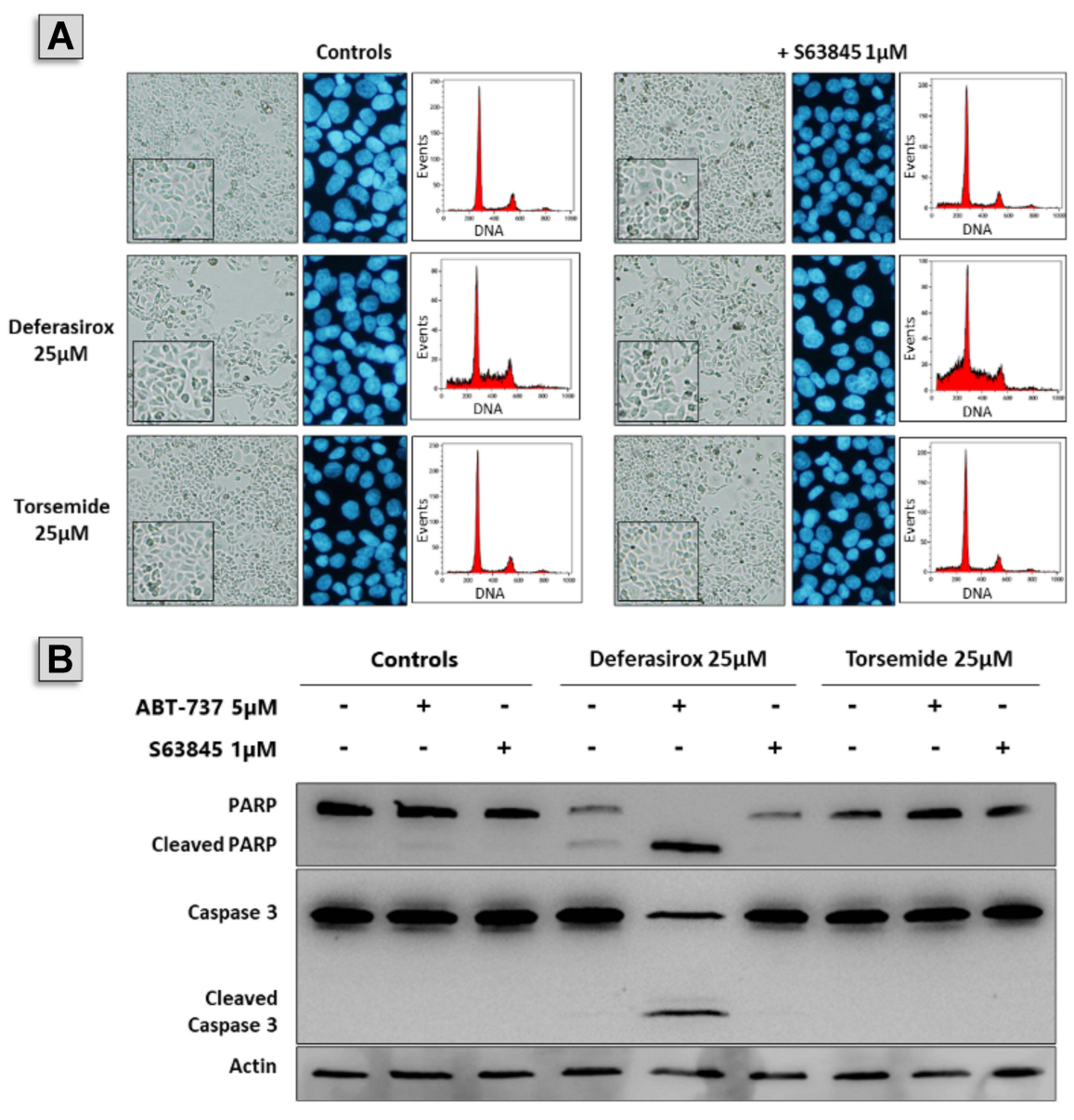

Figure 10 Effect of Deferasirox and Torsemide in combination with ABT-737 or S63845 on apoptosis on IGROVI-RIO cell line. Twenty-four hours after seeding, IGROVIRI 0 ovarian cancer cells were exposed to $25 \mu$ M Deferasirox or Torsemide for $48 \mathrm{~h}$ in absence or presence of $5 \mu$ M ABT-737 or I $\mu$ M S63845. (A) Effects of Deferasirox or Torsemide on cellular morphology, DNA content and nuclear morphology. (B) PARP and caspase 3 cleavage was assessed by Western blot in order to visualize apoptosis induction.

conservation of the intramolecular H-bond in Deferasirox was also regarded during the selection. The selected poses for both ligands corresponded to cluster 1 centroid and cluster 2 centroid, two most energy-favorable clusters from docking, and they are shown in Figure 11.

In the selected poses, Torsemide interacted with Mcl-1 through hydrophobic contacts occurring principally in Mcl-1 P2 pocket and through a $\pi$-stacking of its phenol ring with Phe270 (Figure 11C and D). In pose 1, Torsemide interacted with Leu235 and Leu267 while in pose2 the hydrophobic contacts were more numerous (Phe228, Val253, Phe254, Asp256, Thr266 and Leu267). The Val253, Phe270, Leu267, residues forming $\mathrm{P} 2$ pocket, were identified as strongly perturbed in NMR analysis. No H-bond was detected between Torsemide and Mcl-1 in both poses, corroborating the experimentally observed weak affinity of Torsemide.

In selected Deferasirox pose1 (Figure 11A), the one phenol ring of Deferasirox was placed in the Mcl-1 P2 pocket, in agreement with NMR observations, while the benzoate anion was oriented towards Arg263, one of the residues detected as strong perturbed during the NMR experience. Deferasirox interacted with the Arg263 via $\pi$ cation interaction between the guanidinium group and the phenol ring, and an H-bond with the triazole nitrogen ring. Stabilizing hydrophobic contacts were also formed with Val253, Phe254 and Leu267. Val 253 and Leu 267 were also strongly perturbed in NMR study.

Concerning the second binding mode (pose2 Figure 11B), it was the other phenol ring that was positioned in the Mcl-1 P2 pocket and the benzoate anion was returned to the opposite site of the Mcl-1 binding groove, towards Lys234, another residue revealed as strong perturbed in NMR study. Deferasirox established a salt bridge with Lys234, an H-bond with Arg263 and a $\pi$ - $\pi$ stacking interaction with Phe270. Hydrophobic contacts were made with Val253. 


\section{Deferasirox}

A

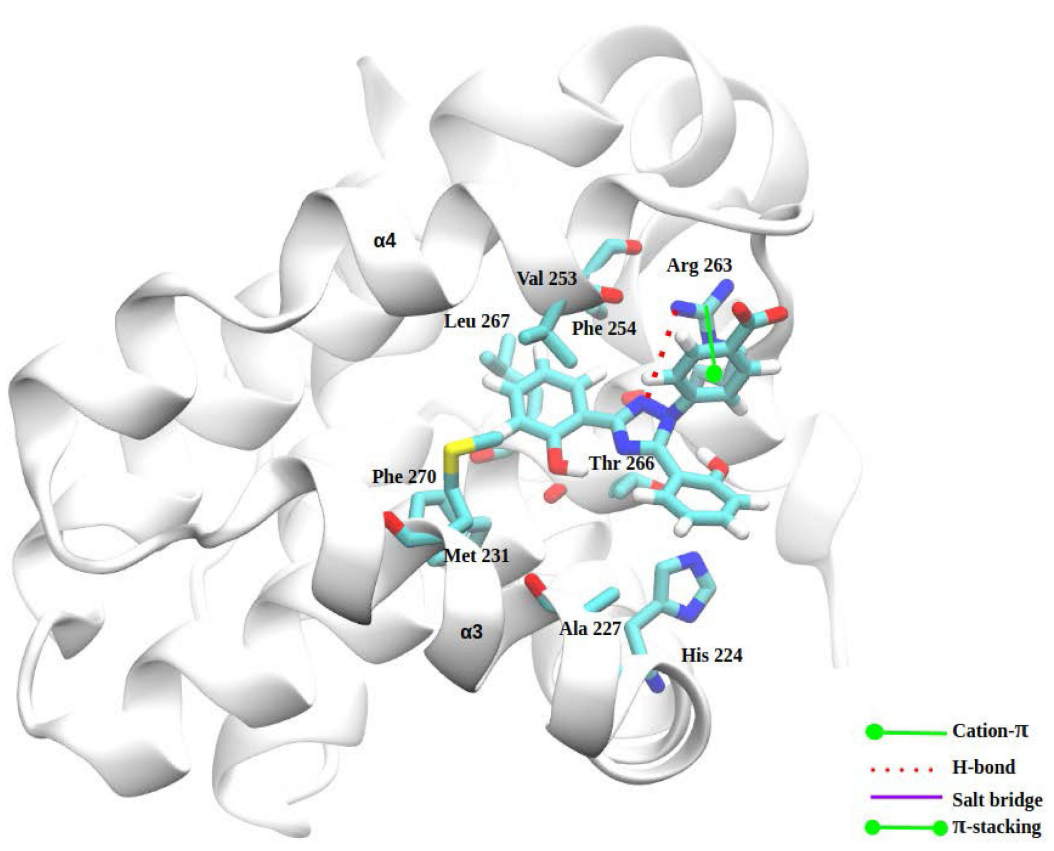

B

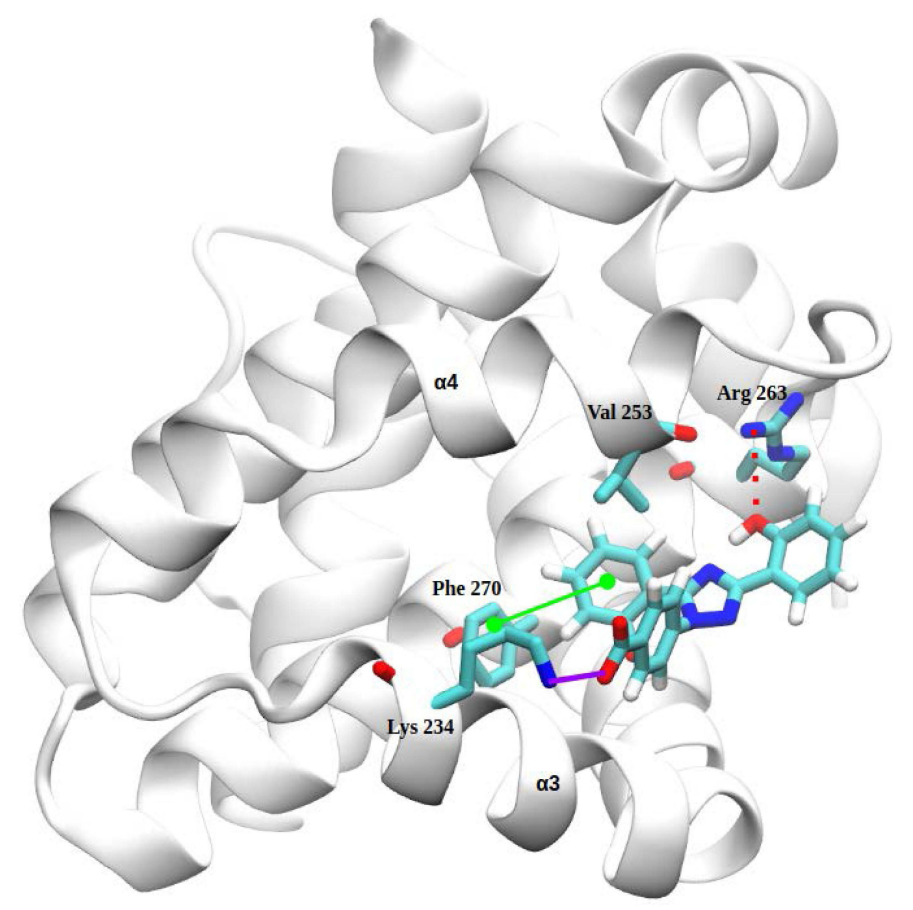

Figure I I Continued.

Binding modes extracted from molecular docking are suitable to establish the first guess, but the dynamical nature and stability of the noncovalent interactions are not considered. Hence, we complemented our analysis by including the dynamical nature of the noncovalent interactions during the MD simulations. As shown in Figure 12 the primarily predicted interactions varied during the MD simulations.

During the dynamics of Torsemide pose1 and pose2, the Torsemide remained fixed by its phenol ring in the 


\section{Torsemide}

C

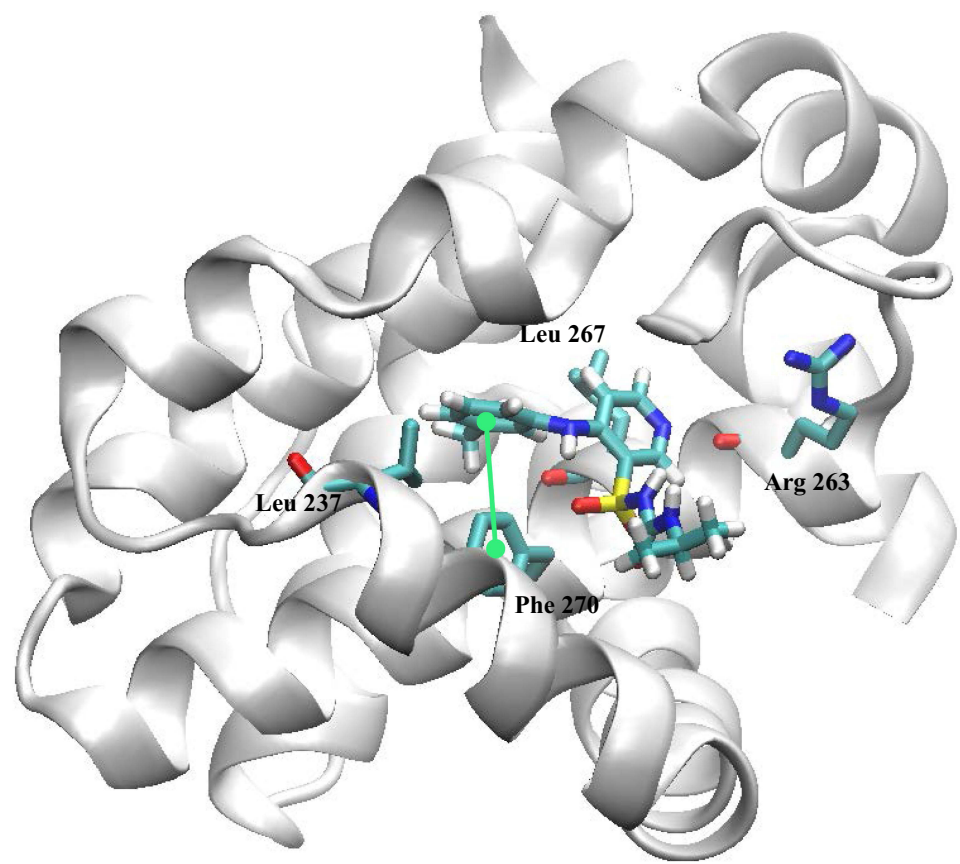

D

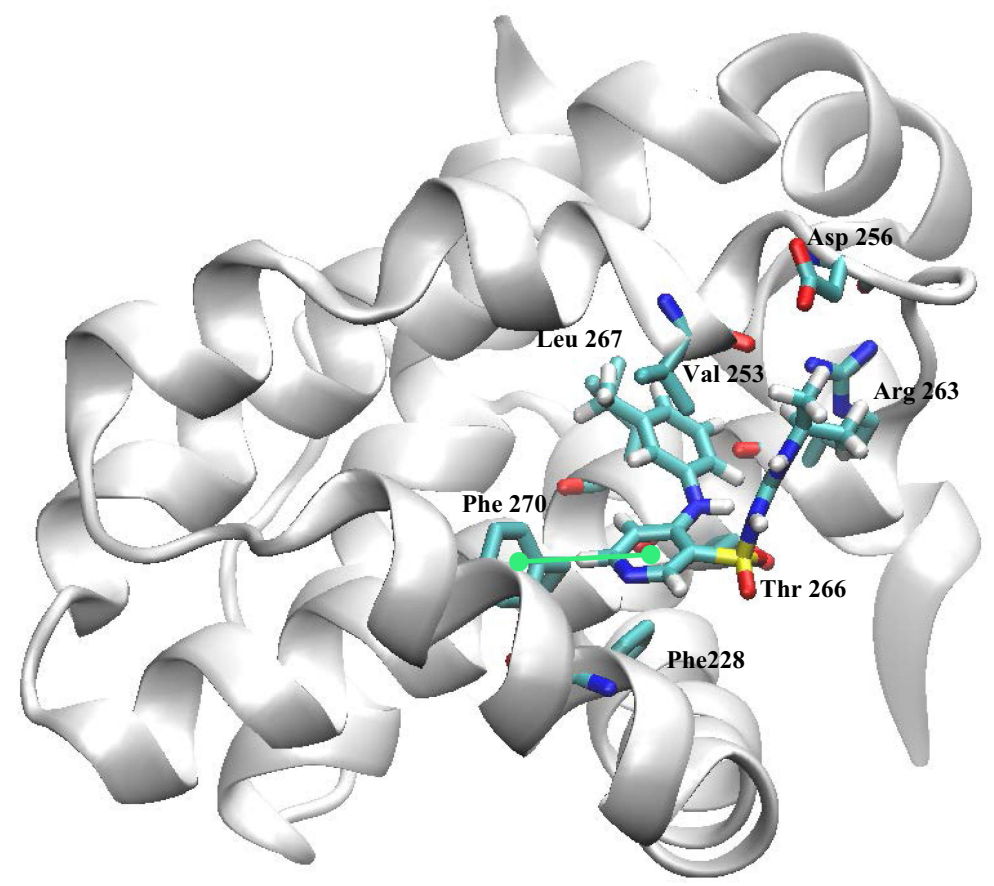

Figure II Docking binding poses of Deferasirox in Mcl-I; (A) Pose I; (B) Pose 2 and of Torsemide; (C) Pose I and (D) Pose 2.

Mcl-1 P2 pocket, its aliphatic chain was very flexible and at the same time came close to Arg263 or His224 to form an H-bond. However, it failed to establish a stable interaction with Mcl-1 along the dynamics (Figure 12A and B). To be able to establish electrostatic interactions with Arg263 or His224 Torsemide must come out of the 


\section{Torsemide}
A

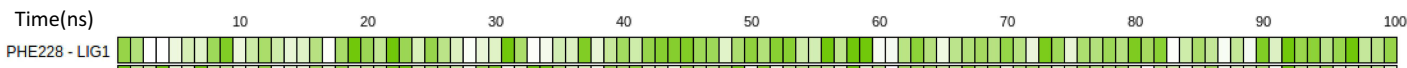

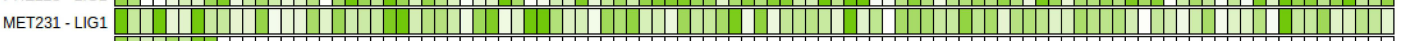 LEU235 - LIG1 \begin{tabular}{l|l|l|l|l|}
\hline MET250 - LIG1 \\
\hline \hline
\end{tabular}

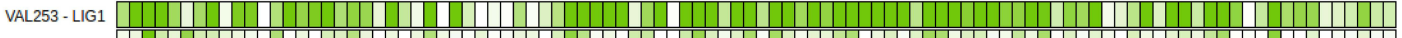

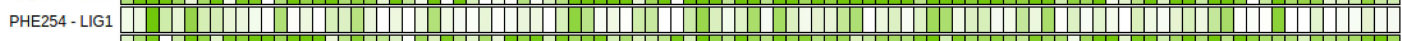

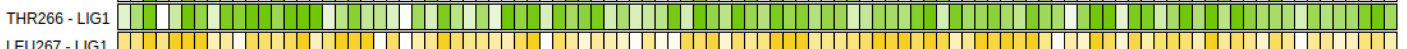 LEU267 - LIG1 - LG1

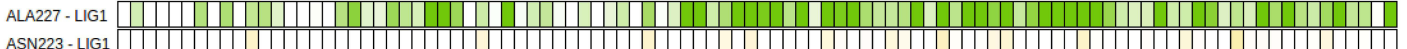

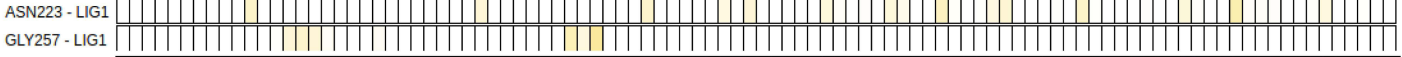

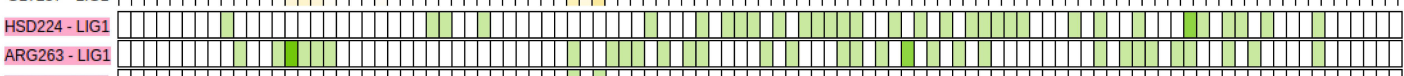

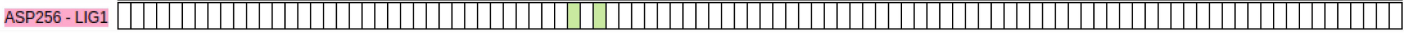
Time(ns)
10
20
PHE228 - LIG1
MET231 - LIG1
MET250 - LIG1 DID
VAL253 - LIG1
PHE254 - LIG1

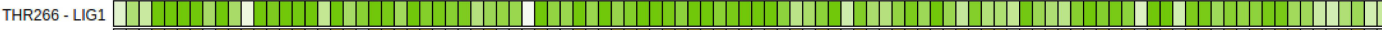

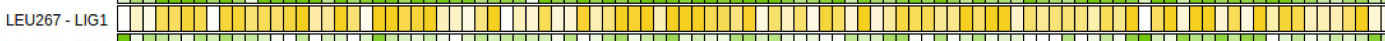
\begin{tabular}{l|l|l|l|l|l|l|l|l|l|l|l|l|l|l|l|l|l|l|l|l|l|l|l|l|l|} 
PHE270 - LIG1 \\
ASN223 - LIG1
\end{tabular}

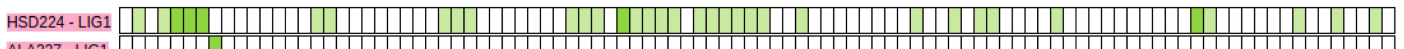

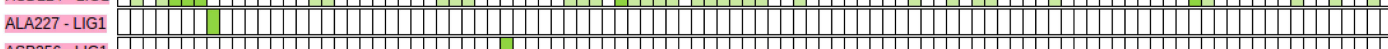

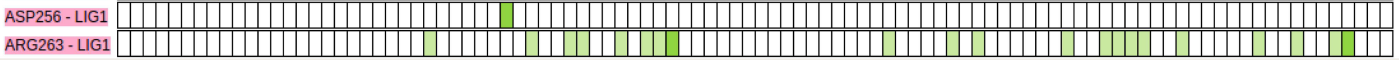

B

\section{Deferasirox}

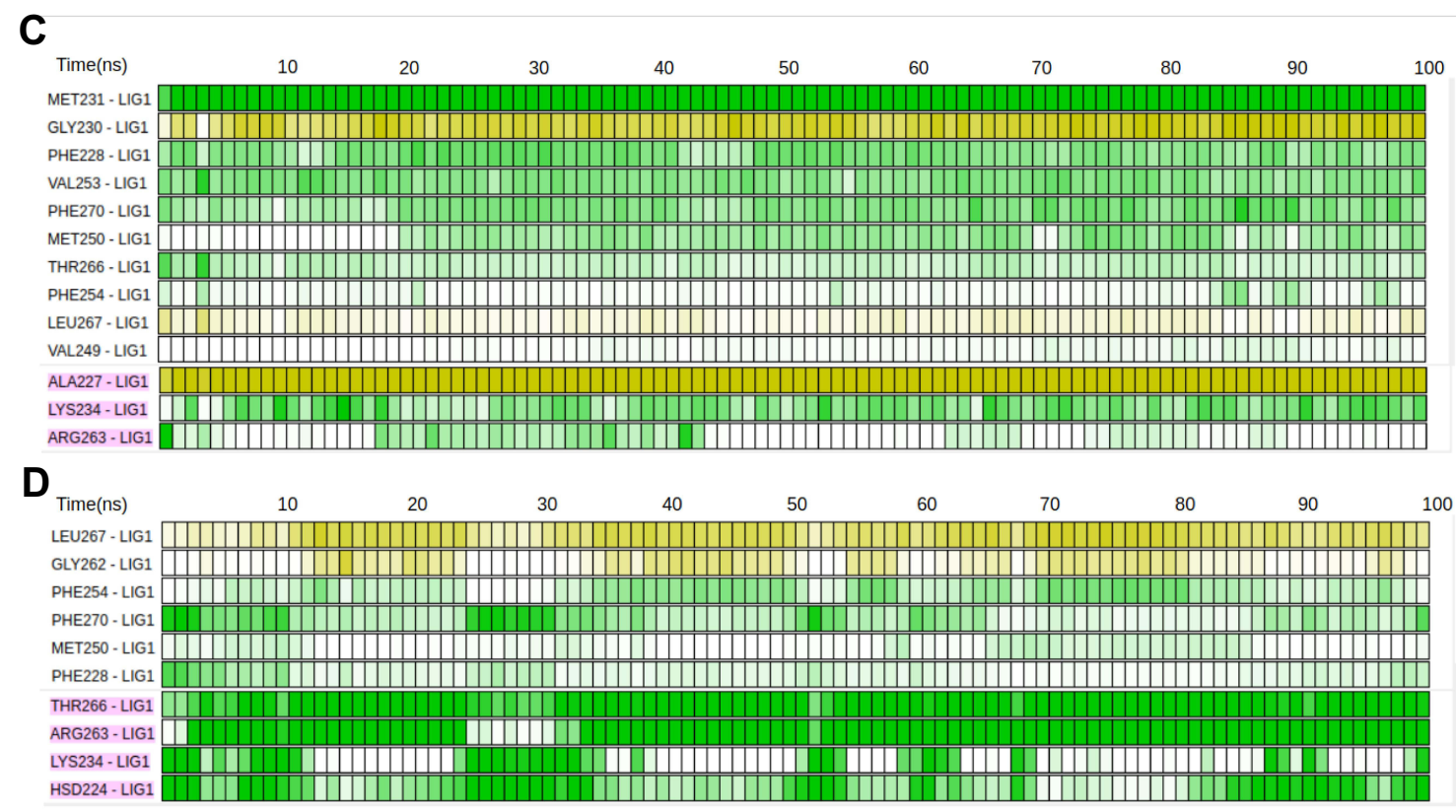

Figure 12 Key interacting residues (sorted using the mean lifetime of the interaction) and dynamical evolution of noncovalent interactions for MD simulation of pose I (A) and pose 2 (B) of Torsemide and pose I (C) and pose 2 (D) of Deferasirox complexes. Each cell accounts for Ins; yellow cell indicates an interaction through the protein's backbone; green cell indicates an interaction through the sidechain. A color gradient was used in order to account for the interaction distance. Hydrogen bonds and electrostatic interactions are colored in pink. 
cavity and hydrophobic contacts with P2 pocket decreased (Figure S4 and Supplementary Video 1). 12 residues-maintained hydrophobic contacts with Torsemide in pose1 during the simulation and the main contributing residues were Val 253, Thr266, Leu267 (main chain) and Phe270. For pose 2 binding mode, 9 residues maintained the contact with Torsemide and the main contributing residues were Val253, Thr266 and Leu267 (main chain). As the Torsemide aliphatic chain did not establish a stable H-bond interaction, the detected electrostatic contacts were punctual. Our dynamic study therefore suggests that Torsemide is able to simultaneously establish the H-bond interaction with $\operatorname{Arg} 263$ (or other charged residue) and fully occupy the P2 pocket, which can explain its weak affinity (Figure 13A). The main anchoring point of Torsemide in Mcl-1 is the P2 pocket according to the modelling results, which is in agreement with NMR observations. The P2 pocket residues were observed mostly among most perturbed residues highlighted by NMR. Then, a propagation effect could explain the detected perturbation of residues in $\mathrm{P} 2$ pocket vicinity and that of Arg263. The simulations suggest that the observed perturbation of P1 pocket residues (Leu232, Leu235 and Lys238) is related to fluctuation of $\alpha 3-\alpha 4$ loop then the direct Torsemide binding effect.

Moreover, the NMR data suggested an interaction with Gly219, Val220 and Val216, part of the P4 pocket, for both ligands. This observation was also highlighted from similar NMR data reported by Mady et al, ${ }^{68}$ for a set of Mcl-1 inhibitors. These interactions at the $\mathrm{P} 4$ pocket might rise from a non-direct effect due to the presence of aromatic rings (ring-current effect) as we have previously discussed for Pyridoclax ${ }^{36}$ for instance. Another explanation might be derived from two steps binding process: the ligands form a pre-encounter complex at the P4 pocket (Val216 and Gly219) in the first place and shift afterwards to the $\mathrm{P} 2 / \mathrm{P} 3$ pocket interface as a second step. This two-step binding process was proposed for $\mathrm{Bcl}-\mathrm{x}_{\mathrm{L}}$ (another antiapoptotic protein) and reported by the work of Krishnamoorthy et al, ${ }^{69}$ using NMR chemical shift data and J-Surface mapping.

For pose 1 the Deferasirox turned on itself at the beginning of the dynamics bringing the carboxylate group towards Lys234 (Figure S5 and Supplementary Video 2). 13 residues-maintained contacts with Deferasirox during the MD simulation (Figure 12C and D). Among them, the main contributing residues to the interaction with Deferasirox were Met231 and Gly230 (backbone), Phe228, Val253 through hydrophobic contacts. Hydrogen bonds with Arg263, Lys234 side chains, as well as an electrostatic interaction with Ala227 (backbone) were noticed.

For pose 2 binding mode, the Deferasirox oscillated around the pose from docking during the MD simulation and 10 residues maintained the contact with it. The residues: Leu267 (backbone), Gly262 (backbone), Phe254, Phe270 established hydrophobic contacts, while Thr266, His224, Arg263 and Lys234 side chains established hydrogen bonds.

Interestingly, the presence of an intramolecular H-bond in Deferasirox has been preserved along both dynamics (Figure S6). The contacts established by Deferasirox were more preserved and stronger along the dynamics compared to Torsemide (Figure 12). The Deferasirox electrostatic interactions were more important in agreement with its observed higher affinity.

A more detailed description of Deferasirox dynamics with Mcl-1 is provided in Supplementary Video 2. Overall, Deferasirox interacts with Mcl-1's binding groove by establishing contacts with $\mathrm{P} 2$ and $\mathrm{P} 3$ pockets (Figure 13B). The most interacting residues in pose 2 MD simulation are in an overall good agreement with the most perturbed residues determined by NMR chemical shifts.

Combining the MD simulations and NMR chemical shifts, we propose the following binding mode for Deferasirox with Mcl-1 protein (Figure 13B). As discussed above, Deferasirox interacts directly with the $\mathrm{P} 2 / \mathrm{P} 3$ pockets establishing noncovalent interactions (hydrophobic interactions). The observed perturbation of $\mathrm{P} 1$ pocket residues (Leu235 and Asp236) is due to the interaction with the Lys234 through a salt bridge (propagation effect). Finally, the perturbation of Val220 and Val216 residues, part of the P4 pocket could be due to either the wellknown ring-current effect for aromatic rings (mediated by the presence of the aromatic ring nearby the $\mathrm{P} 4$ pocket) or a result of two steps binding mechanism, not highlighted for moment in the docking.

\section{Conclusion}

In this study, the interaction between Mcl-1 and five drugs already used for other therapeutic indications and highlighted in literature as potential Mcl-1 inhibitor (Oxcarbazepine, Deferasirox, Risperidone, Lenalidomide and Torsemide) were experimentally characterized by NMR. 


\section{A}

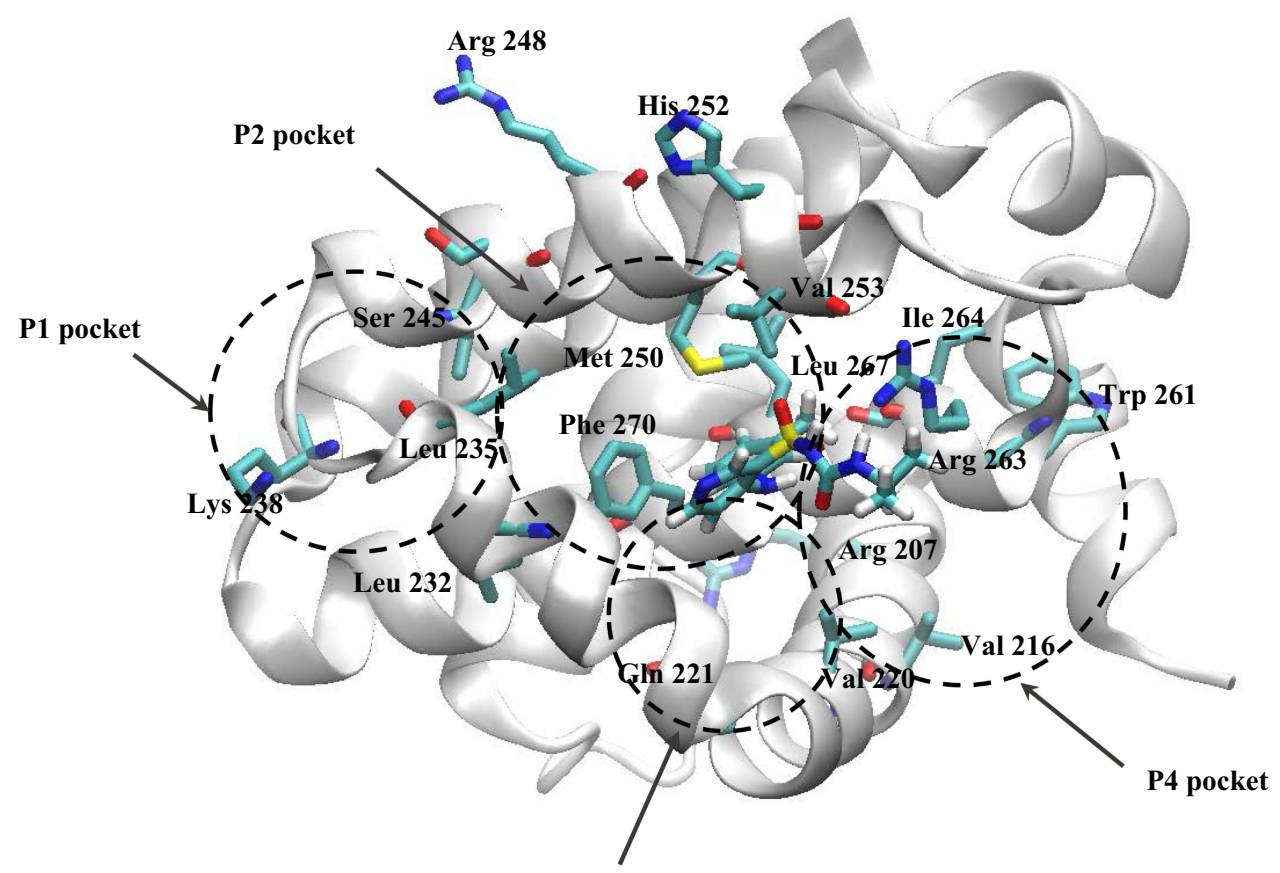

$P 3$ pocket

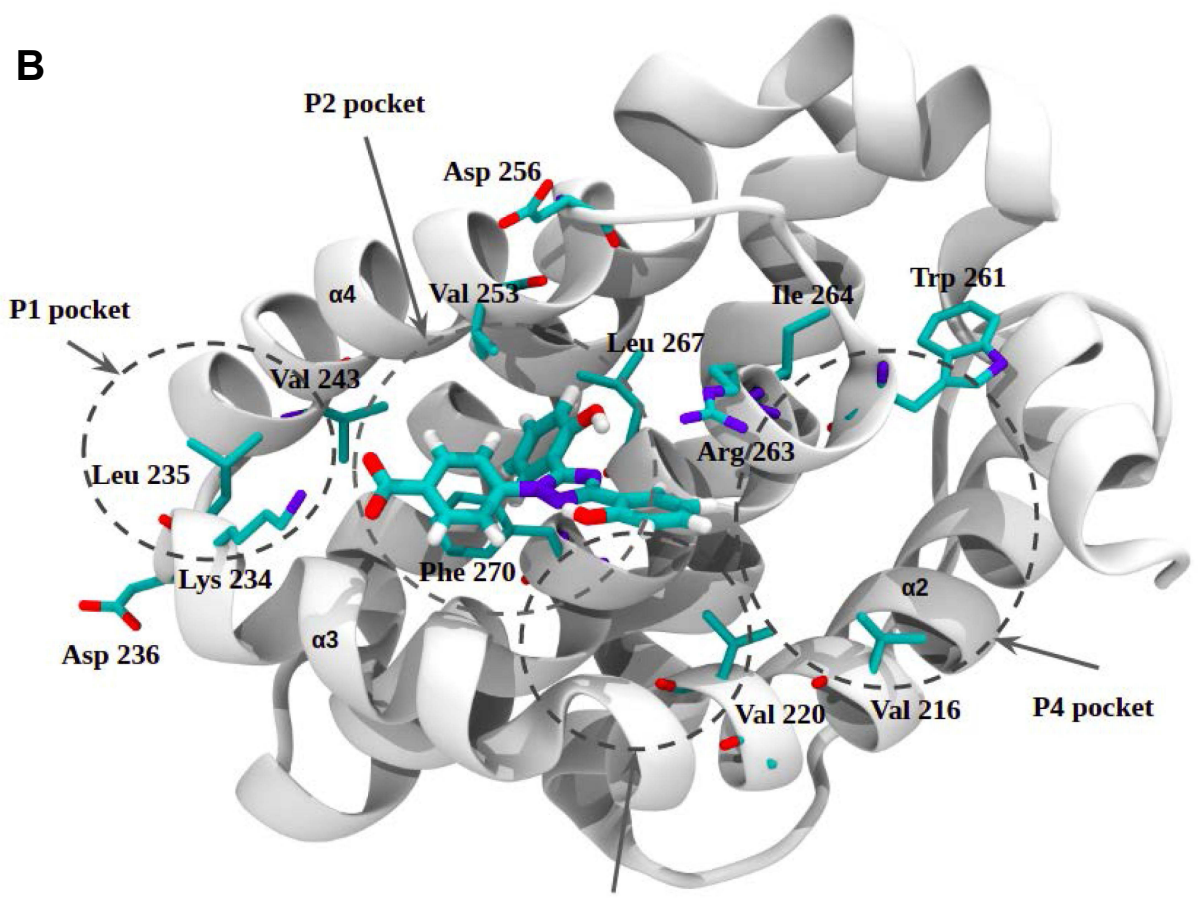

P3 pocket

Figure 13 (A) Torsemide-Mcl-I binding mode (end of pose I simulation). (B) Deferasirox-Mcl-I binding mode (end of the pose 2 simulation). Mcl-I perturbed residues by Torsemide and Deferasirox from NMR study are shown in licorice representation (residue CCSP $>0.10$ ppm). 
NMR analysis showed that only two of the five tested molecules, Torsemide and Deferasirox, interact with Mcl1. The interaction sites evidenced by NMR for these two drugs are mainly centered on around the P2 hydrophobic pocket, Deferasirox showing a stronger affinity for Mcl-1 $\left(\mathrm{K}_{\mathrm{D}} \sim 300 \mu \mathrm{M}\right)$ than Torsemide $\left(\mathrm{K}_{\mathrm{D}} \sim 1 \mathrm{mM}\right)$.

Biological assays confirmed that Deferasirox was able to inhibit Mcl-1 efficiently and selectively in a dose and time-dependent manner with optimal dose of $25 \mu \mathrm{M}$ for a $48 \mathrm{~h}$ exposure treatment. The biological activity of Torsemide was lower as compared to the Deferasirox one since a $50 \mu \mathrm{M}$ concentration was required to observe similar effects.

FPA competition assays confirmed that Deferasirox was able to disrupt interactions between Mcl-1 with Bid, a pro-apoptotic partner of Mcl-1. An inhibition close to $60 \%$ (at $600 \mu \mathrm{M}$ ) was observed and $\mathrm{IC}_{50}$ and $\mathrm{K}_{\mathrm{i}}$ values of $368 \pm 45 \mu \mathrm{M}$ and $83 \pm 10 \mu \mathrm{M}$, respectively, were obtained for Deferasirox. A weaker inhibition close to $20 \%$ (at 600 $\mu \mathrm{M})$ was observed with Torsemide.

Finally, combining the MD simulations and NMR chemical shift perturbation analysis, we proposed a binding mode for Deferasirox with Mcl-1 protein. Deferasirox interacts directly with the $\mathrm{P} 2 / \mathrm{P} 3$ pockets, as well as almost all synthetic Mcl-1 inhibitors, establishing noncovalent hydrophobic interactions. However, contrary to most synthetic Mcl-1 inhibitors, Deferasirox interacts through a salt bridge with Lys234 and not with Arg263. The Torsemide binds to P2 pocket and our simulations suggest that it is able to simultaneously interact with $\mathrm{P} 2$ pocket and form an H-bond with Arg263. It oscillates between these two binding spots.

In conclusion, this work evidenced the complementarity of computational and experimental approaches in drug repositioning. Combining both approaches, we showed that Deferasirox could be a potential candidate for drug repositioning as Mcl-1 inhibitor.

\section{Acknowledgments}

This work has been partially supported by Normandie Université (NU), the Région Normandie, the Centre National de la Recherche Scientifique (CNRS), Université de Rouen Normandie (URN), INSA Rouen Normandie, Labex SynOrg (ANR-11-LABX-0029), Innovation Chimie Carnot (I2C). Part of this work was performed using computing resources of CRIANN (Normandy, France) as well as the European Community (FEDER) for the molecular modeling software.
We thank Christophe Denoyelle and Emilie Brotin from ImpedanCELL platform for real-time imaging experiments. The IncuCyte S3 device was acquired thanks to the support of the French State and the 'Normandy County Council' (Contrat de Plan Etat Région - CPER INNOVONS). This work was also supported by the "Conseil Régional de Basse Normandie" and European Union (The ONCOTHERA European project "Normandy Network for innovative therapeutics in oncology" which is co-funded by the Normandy County Council, the European Union within the framework of the Operational Programme ERDF/ESF 2014-2020), by the French State (CPER Innovons 2), the University of Caen Normandie and Inserm. HP is a recipient of a doctoral fellowship from the French Ministry for Higher Education and Research.

\section{Author Contributions}

ABA, ASVC, MS and HO conceived the studies; ABA and MS performed NMR studies; DR and LC have carried out the production and purification of the labeled Mcl-1 ${ }^{172}$ ${ }^{-327}$ domain; HP, LW and LP have carried out bioassays; MJ carried out FPA experiments; MB, GC and JSO performed docking and dynamics simulations; ABA, JSO, and MS wrote the article with corrections from all coauthors. So, all authors made substantial contributions to conception and design, acquisition of data, or analysis and interpretation of data; took part in drafting the article or revising it critically for important intellectual content; agreed to submit to the current journal; gave final approval of the version to be published; and agree to be accountable for all aspects of the work.

\section{Disclosure}

The authors report no conflicts of interest in this work.

\section{References}

1. Ciociola AA, Cohen LB, Kulkarni P, et al. How drugs are developed and approved by the FDA: current process and future directions. Am J Gastroenterol. 2014;109(5):620-623. doi:10.1038/ajg.2013.407

2. Ashburn TT, Thor KB. Drug repositioning: identifying and developing new uses for existing drugs. Nat Rev Drug Discov. 2004;3(8):673-683. doi:10.1038/nrd1468

3. Oprea TI, Bauman JE, Bologa CG, et al. Drug repurposing from an academic perspective. Drug Discov Today Ther Strateg. 2011;8(34):61-69. doi:10.1016/j.ddstr.2011.10.002

4. Langedijk J, Mantel-Teeuwisse AK, Slijkerman DS, Schutjens MHDB. Drug repositioning and repurposing: terminology and definitions in literature. Drug Discov Today. 2015;20(8):1027-1034. doi:10.1016/ j.drudis.2015.05.001

5. Pushpakom S, Iorio F, Eyers PA, et al. Drug repurposing: progress, challenges and recommendations. Nat Rev Drug Discov. 2019;18 (1):41-58. doi:10.1038/nrd.2018.168 
6. Hurle MR, Yang L, Xie Q, Rajpal DK, Sanseau P, Agarwal P. Computational drug repositioning: from data to therapeutics. Clin Pharmacol Ther. 2013;93(4):335-341. doi:10.1038/clpt.2013.1

7. Shim JS, Liu JO. Recent advances in drug repositioning for the discovery of new anticancer drugs. Int $J$ of Biol Sci. 2014;10 (7):654-663. doi:10.7150/ijbs.9224

8. Luo H, Li M, Yang M, Wu F-X, Li Y, Wang J. Biomedical data and computational models for drug repositioning: a comprehensive review. Brief Bioinform. 2021;22(2):1604-1619. doi:10.1093/bib/ bbz176

9. Hernandez JJ, Pryszlak M, Smith L, et al. Giving drugs a second chance: overcoming regulatory and financial hurdles in repurposing approved drugs as cancer therapeutics. Front Oncol. 2017;7:273. doi:10.3389/fonc.2017.00273

10. Glantz-Gashai Y, Meirson T, Reuveni E, Samson AO. Virtual screening for potential inhibitors of Mcl-1 conformations sampled by normal modes, molecular dynamics, and nuclear magnetic resonance. Drug Des Devel Ther. 2017;11:1803-1813. doi:10.2147/DDDT. S133127

11. Gelinas C, White E. BH3-only proteins in control: specificity regulates MCL-1 and BAK-mediated apoptosis. Genes Dev. 2005;19 (11):1263-1268. doi:10.1101/gad.1326205

12. Shore GC, Warr MR. Unique biology of Mcl-1: therapeutic opportunities in cancer. Curr Mol Med. 2008;8(2):138-147. doi:10.2174/ 156652408783769580

13. Beroukhim R, Mermel CH, Porter D, et al. The landscape of somatic copy-number alteration across human cancers. Nature. 2010;463 (7283):899-905. doi:10.1038/nature08822

14. Beekman AM, Howell LA. Small-molecule and peptide inhibitors of the pro-survival protein Mcl-1. ChemMedChem. 2015;11(8):802813. doi:10.1002/cmdc. 201500497

15. Belmar J, Fesik SW. Small molecule Mcl-1 inhibitors for the treatment of cancer. Pharmacol Ther. 2015;145:76-84. doi:10.1016/j. pharmthera.2014.08.003

16. Abid M, Sonawane YA, Contreras JI, Rana S, Natarajan A. Recent advances in cancer drug development: targeting induced myeloid cell leukemia-1 (Mcl-1) differentiation protein. Curr Med Chem. 2017;24 (40):4488-4514. doi:10.2174/0929867324666170912092659

17. Ashkenazi A, Fairbrother WJ, Leverson JD, Souers AJ. From basic apoptosis discoveries to advanced selective BCL-2 family inhibitors. Nat Rev Drug Discov. 2017;16(4):273-284. doi:10.1038/ $\operatorname{nrd} .2016 .253$

18. Wan Y, Dai N, Tang Z, Fang H. Small-molecule Mcl-1 inhibitors: emerging anti-tumor agents. Eur J Med Chem. 2018;146:471-482. doi:10.1016/j.ejmech.2018.01.076

19. Hird AW, Tron AE. Recent advances in the development of Mcl-1 inhibitors for cancer therapy. Pharmacol Ther. 2019;198:59-67. doi:10.1016/j.pharmthera.2019.02.007

20. Senichkin VV, Streletskaia AY, Gorbunova AS, Zhivotovsky B, Kopeina GS. Saga of Mcl-1: regulation from transcription to degradation. Cell Death Differ. 2020;27(2):405-419. doi:10.1038/s41418019-0486-3

21. Rasmussen ML, Taneja N, Neininger AC, et al. MCL-1 inhibition by selective BH3 mimetics disrupts mitochondrial dynamics causing loss of viability and functionality of human cardiomyocytes. iScience. 2020;23(4):101015. doi:10.1016/j.isci.2020.101015

22. Ball S, Borthakur G. Apoptosis targeted therapies in acute myeloid leukemia: an update. Expert Rev Hematol. 2020;13(12):1373-1386. doi:10.1080/17474086.2020.1852923

23. Wood KC. Overcoming MCL-1-driven adaptive resistance to targeted therapies. Nat Commun. 2020;11(1):531. doi:10.1038/s41467-02014392-z

24. Wishart DS, Knox C, Guo AC, et al. DrugBank: a knowledgebase for drugs, drug actions and drug targets. Nucleic Acids Res. 2008;36 (suppl 1):D901-D906. doi:10.1093/nar/gkm958
25. Yang LPH, Keam SJ, Keating GM. Deferasirox: a review of its use in the management of transfusional chronic iron overload. Drugs. 2007;67(15):2211-2230. doi:10.2165/00003495-200767150-00007

26. Kamihara Y, Takada K, Sato T, et al. The iron chelator deferasirox induces apoptosis by targeting oncogenic Pyk $2 / \beta$-catenin signaling in human multiple myeloma. Oncotarget. 2016;7:39. doi:10.18632/ oncotarget. 11830

27. Möller HJ. Risperidone: a review. Expert Opin on Pharmacother. 2005;6(5):803-818. doi:10.1517/14656566.6.5.803

28. Degterev A, Lugovskoy A, Cardone M, et al. Identification of smallmolecule inhibitors of interaction between the $\mathrm{BH} 3$ domain and $\mathrm{Bcl}-$ xL. Nat Cell Biol. 2001;3(2):173-182. doi:10.1038/35055085

29. Maier SK, Hammond JM. Role of lenalidomide in the treatment of multiple myeloma and myelodysplastic syndrome. Ann Pharmacother. 2006;40(2):286-289. doi:10.1345/aph.1G170

30. Mark TM, Bowman IA, Rossi AC, et al. Thalidomide, clarithromycin, lenalidomide and dexamethasone therapy in newly diagnosed, symptomatic multiple myeloma. Leuk Lymphoma. 2014;55 (12):2842-2849. doi:10.3109/10428194.2014.896005

31. Friedel HA, Buckley MM-T. Torasemide: a review of its pharmacological properties and therapeutic potential. Drugs. 1991;41(1):81103. doi:10.2165/00003495-199141010-00008

32. van Delft MF, Wei AH, Mason KD, et al. The BH3 mimetic ABT-737 targets selective $\mathrm{Bcl}-2$ proteins and efficiently induces apoptosis via Bak/Bax if Mcl-1 is neutralized. Cancer Cell. 2006;10(5):389-399. doi:10.1016/j.ccr.2006.08.027

33. Meyer B, Peters T. NMR spectroscopy techniques for screening and identifying ligand binding to protein receptors. Angew Chem Int Ed Engl. 2003;42(8):864-890. doi:10.1002/anie.200390233

34. Cala $\mathrm{O}$, Guillière $\mathrm{F}$, Krimm I. NMR-based analysis of protein-ligand interactions. Anal Bioanal Chem. 2014;406(4):943-956. doi:10.1007/ s00216-013-6931-0

35. Orts J, Gossert AD. Structure determination of protein-ligand complexes by NMR in solution. Methods. 2018;138-139:3-25. doi:10.1016/j.ymeth.2018.01.019

36. Bourafai-Aziez A, Sebban M, Benabderrahmane M, et al. Binding mode of Pyridoclax to myeloid cell leukemia-1 (Mcl-1) revealed by nuclear magnetic resonance spectroscopy, docking and molecular dynamics approaches. J Biomol Struct Dyn. 2020;38(14):41624178. doi:10.1080/07391102.2019.1680434

37. Mori S, Abeygunawardana C, Johnson MO, Vanzijl PCM. Improved sensitivity of HSQC spectra of exchanging protons at short interscan delays using a new fast HSQC (FHSQC) detection scheme that avoids water saturation. J Magn Reson B. 1995;108(1):94-98. doi:10.1006/jmrb.1995.1109

38. Marion D, Wüthrich K. Application of phase sensitive two-dimensional correlated spectroscopy (COSY) for measurements of $1 \mathrm{H}-1 \mathrm{H}$ spin-spin coupling constants in proteins. Biochem Biophys Res Commun. 1983;113(3):967-974. doi:10.1016/0006-291X(83)91093-8

39. Lee W, Tonelli M, Markley JL. NMRFAM-SPARKY: enhanced software for biomolecular NMR spectroscopy. Bioinformatics. 2015;31 (8):1325-1327. doi:10.1093/bioinformatics/btu830

40. Skinner SP, Fogh RH, Boucher W, Ragan TJ, Mureddu LG, Vuister GW. CcpNmr AnalysisAssign: a flexible platform for integrated NMR analysis. J Biomol NMR. 2016;66(2):111-124. doi:10.1007/ s10858-016-0060-y

41. Waudby CA, Ramos A, Cabrita LD, Christodoulou J. Two-dimensional NMR lineshape analysis. Sci Rep. 2016;6:24826. doi:10.1038/ srep24826

42. Williamson MP. Using chemical shift perturbation to characterise ligand binding. Prog Nucl Magn Reson Spectrosc. 2013;73:1-16. doi:10.1016/j.pnmrs.2013.02.001

43. Poulain L, Lincet H, Duigou F, et al. Acquisition of chemoresistance in a human ovarian carcinoma cell is linked to a defect in cell cycle control. Int J Cancer. 1998;78(4):454-463. doi:10.1002/(sici)10970215(19981109)78:4<454::aid-ijc11>3.0.co;2-6 
44. Nikolovska-Coleska Z, Wang R, Fang X, et al. Development and optimization of a binding assay for the XIAP BIR3 domain using fluorescence polarization. Anal Biochem. 2004;332:261. doi:10.1016/ j.ab.2004.05.055

45. Tanaka Y, Aikawa K, Nishida G, et al. Discovery of potent Mcl-1/ Bcl-xL dual inhibitors by using a hybridization strategy based on structural analysis of target proteins. J Med Chem. 2013;56 (23):9635-9645. doi:10.1021/jm401170c

46. Sastry GM, Adzhigirey M, Day T, Annabhimoju R, Sherman W. Protein and ligand preparation: parameters, protocols, and influence on virtual screening enrichments. J Comput Aided Mol Des. 2013;27 (3):221-234. doi:10.1007/s10822-013-9644-8

47. Fun H-K, Chantrapromma S, Dayananda AS, Yathirajan HS, Thomas S. 4-[3,5-Bis(2-hy-droxy-phen-yl)-1H-1,2,4-triazol-1-yl]benzoic acid dimethyl-formamide monosolvate. Acta Crystallogr Sect E Struct Rep Online. 2012;68(Pt 3):o792-o793. doi:10.1107/S160053681 2005806

48. Groom CR, Bruno IJ, Lightfoot MP, Ward SC. The Cambridge structural database. Acta Cryst B. 2016;72(2):171-179. doi:10.1107/ S2052520616003954

49. Gutman A, Etinger M, Goldring D, Pertzikov B, Nisnevich G. CCDC 795036: experimental crystal structure determination; 2011.

50. Grosdidier A, Zoete V, Michielin O. Fast docking using the CHARMM force field with EADock DSS. J Comput Chem. 2011;32(10):2149-2159. doi:10.1002/jcc.21797

51. Grosdidier A, Zoete V, Michielin O. SwissDock, a protein-small molecule docking web service based on EADock DSS. Nucleic Acids Res. 2011;39:W270-W277. doi:10.1093/nar/gkr366

52. Zoete V, Grosdidier A, Cuendet M, Michielin O. Use of the FACTS solvation model for protein-ligand docking calculations. Application to EADock. J Mol Recognit. 2010;23(5):457-461. doi:10.1002/ jmr. 1012

53. Phillips JC, Braun R, Wang W, et al. Scalable molecular dynamics with NAMD. J Comput Chem. 2005;26(16):1781-1802. doi:10.1002/ jcc. 20289

54. Huang J, MacKerell AD. CHARMM36 all-atom additive protein force field: validation based on comparison to NMR data. J Comput Chem. 2013;34(25):2135-2145. doi:10.1002/jcc.23354

55. Huang J, Rauscher S, Nawrocki G, et al. CHARMM36m: an improved force field for folded and intrinsically disordered proteins. Nat Methods. 2017;14(1):71-73. doi:10.1038/nmeth.4067

56. Vanommeslaeghe K, Hatcher E, Acharya C, et al. CHARMM general force field: a force field for drug-like molecules compatible with the CHARMM all-atom additive biological force fields. J Comput Chem. 2009;31(4):671-690. doi:10.1002/jcc.21367

57. Lee J, Cheng X, Swails JM, et al. CHARMM-GUI input generator for NAMD, GROMACS, AMBER, OpenMM, and CHARMM/OpenMM simulations using the CHARMM36 additive force field. $J$ Chem Theory Comput. 2016;12(1):405-413. doi:10.1021/acs.jctc.5b00935
58. Jorgensen WL, Jenson C. Temperature dependence of TIP3P, SPC, and TIP4P water from NPT Monte Carlo simulations: seeking temperatures of maximum density. $J$ Comput Chem. 1998;19(10):1179-1186. doi:10.1002/(SICI)1096-987X(19980730)19:10<1179::AID-JCC6>3.0. CO;2-J

59. Darden T, York D, Pedersen L. Particle mesh Ewald: an $\mathrm{N} \cdot \log (\mathrm{N})$ method for Ewald sums in large systems. J Chem Phys. 1993;98 (12):10089-10092. doi:10.1063/1.464397

60. Humphrey W, Dalke A, Schulten K. VMD: visual molecular dynamics. J Mol Graph. 1996;14(1):33-38,27-28. doi:10.1016/ 0263-7855(96)00018-5

61. Scheurer M, Rodenkirch P, Siggel M, et al. PyContact: rapid, customizable, and visual analysis of noncovalent interactions in MD simulations. Biophys J. 2018;114(3):577-583. doi:10.1016/j.bpj.2017.12.003

62. Liu G, Poppe L, Aoki K, Yamane H, Lewis J, Szyperski T. Highquality NMR structure of human anti-apoptotic protein domain Mcl-1 (171-327) for cancer drug design. PLoS One. 2014;9(5):e96521. doi:10.1371/journal.pone.0096521

63. Bissantz C, Folkers G, Rognan D. Protein-based virtual screening of chemical databases. 1. evaluation of different docking/scoring combinations. J Med Chem. 2000;43(25):4759-4767. doi:10.1021/jm0010441

64. Benabderrahmane M, Bureau R, Voisin-Chiret AS, Sopkova-de Oliveira Santos J. Insights into Mcl-1 conformational states and allosteric inhibition mechanism from molecular dynamics simulations, enhanced sampling, and pocket crosstalk analysis. J Chem Inf Model. 2020;60(6):3172-3187. doi:10.1021/acs.jcim.0c00315

65. Brotin E, Meryet-Figuière M, Simonin K, et al. Bcl-x L and MCL-1 constitute pertinent targets in ovarian carcinoma and their concomitant inhibition is sufficient to induce apoptosis. Int $J$ Cancer. 2010;126(4):885-895. doi:10.1002/ijc.24787

66. Steinhauser S, Heinz U, Bartholomä M, Weyhermüller T, Nick H, Hegetschweiler K. Complex formation of ICL670 and related ligands with Fe III and Fe II: complex formation of ICL670 and related ligands with Fe III and Fe II. Eur J of Inorg Chem. 2004;2004 (21):4177-4192. doi:10.1002/ejic.200400363

67. Zhang Y, Yang H, Ma H, et al. Excitation wavelength dependent fluorescence of an ESIPT triazole derivative for amine sensing and anti-counterfeiting applications. Angew Chem Int Ed Engl. 2019;58 (26):8773-8778. doi:10.1002/anie.201902890

68. Mady ASA, Liao C, Bajwa N, et al. Discovery of Mcl-1 inhibitors from integrated high throughput and virtual screening. Sci Rep. 2018;8(1):10210. doi:10.1038/s41598-018-27899-9

69. Krishnamoorthy J, Yu VCK, Mok Y-K. Auto-FACE: an NMR based binding site mapping program for fast chemical exchange protein-ligand systems. PLoS One. 2010;5(2):e8943. doi:10.1371/journal.pone.0008943
Drug Design, Development and Therapy

\section{Publish your work in this journal}

Drug Design, Development and Therapy is an international, peerreviewed open-access journal that spans the spectrum of drug design and development through to clinical applications. Clinical outcomes, patient safety, and programs for the development and effective, safe, and sustained use of medicines are a feature of the journal, which has also been accepted for indexing on PubMed Central. The manuscript management system is completely online and includes a very quick and fair peer-review system, which is all easy to use. Visit http://www. dovepress.com/testimonials.php to read real quotes from published authors. 